\title{
Le cerf chez les anciens P'urhépecha du Michoacan (Mexique) : guerre, chasse et sacrifice
}

\section{Brigitte Faugère}

\section{(2) OpenEdition}

12 Journals

Édition électronique

URL : https://journals.openedition.org/jsa/10583

DOI : 10.4000/jsa. 10583

ISSN : 1957-7842

Éditeur

Société des américanistes

\section{Édition imprimée}

Date de publication : 20 décembre 2008

Pagination : 109-142

ISSN : 0037-9174

\section{Référence électronique}

Brigitte Faugère, "Le cerf chez les anciens P'urhépecha du Michoacan (Mexique) : guerre, chasse et sacrifice », Journal de la Société des américanistes [En ligne], 94-2 | 2008, mis en ligne le 10 décembre 2013, consulté le 04 septembre 2022. URL : http://journals.openedition.org/jsa/10583 ; DOI : https:// doi.org/10.4000/jsa. 10583 


\title{
LE CERF CHEZ LES ANCIENS P'URHÉPECHA DU MICHOACAN (MEXIQUE) : GUERRE, CHASSE ET SACRIFICE
}

\author{
Brigitte FAUGÈRE *
}

L'étude de la place des cervidés dans la Relation du Michoacan et dans les vestiges archéologiques permet de montrer l'importance de cet animal dans la mythologie des anciens P'urhépecha (Tarasques) du Michoacan. Le cerf de cette région (Odocoileus virginiamus) a tenu une place importante dans les rites, sa capture pouvant être assimilée à une guerre débouchant sur des pratiques sacrificielles. Associé dans la mythologie tarasque au soleil, au moment où celui-ci se régénère pour surgir à l'Orient, le cerf a aussi joué un rôle particulier pour l'élite au pouvoir au $x{ }^{\mathrm{c}}$ siècle, puisqu'il avait été pris comme animal emblématique par le groupe guerrier dominant. [Mots-clés : cervidé, P'urhépecha, Tarasques, mythologie, guerriers, chasse, sacrifice.]

El venado entre los antiguos p'urhépecha de Michoacán (México) : guerra, caza y sacrificio. El estudio del lugar ocupado por los venados en la Relación de Michoacán y en los vestigios arqueológicos permite mostrar la importancia de este animal dentro de la mitología de los antiguos p'urhépecha (tarascos) de Michoacán. El venado de esta región (Odocoileus virginianus) tuvo un papel importante dentro de la esfera ritual, siendo su captura asimilada a una guerra que desembocaba en sacrificios. Dentro de la mitología, está asociado al sol, en el momento en que se regenera para salir al Oriente. El venado jugo también un papel particular para la elite en el poder a principio del siglo XVI, ya que había sido escogido como un animal emblemático del grupo dominante de los guerreros. [Palabras claves: venado, p'urhépecha, tarascos, mitología, guerreros, caza, sacrificio.]

The deer among the ancient P'urhépecha of Michoacan, Mexico: war, hunting and sacrifice. The study of the place of deers in the Relación de Michoacán and in archaeological remains shows the importance of this animal in ancient Pur'hépecha's mythology. The deer of this region (Odocoileus virginianus) plays a relevant part in the rituals because his capture can be assimilated to a war ending with sacrifices. Within the mythology, it is associated with the sun, when it is regenerating before rising in the east. The deer plays also a particular role for the elite of the 16th century, because it was

* Université Paris 1/UMR 8096, Maison René-Ginouvès (Archéologie et ethnologie), 21 allée de l'université, 92023 Nanterre cedex [brigitte.faugere@univ-paris1.fr].

Journal de la Société des Américanistes, 2008, 94-2, pp. 109-142. (C) Société des Américanistes. 
chosen by the dominant warriors group as an emblematic animal. [Key words : deer, P'urhépecha, Tarascans, mythology, warriors, hunting, sacrifice.]

Au sein du règne animal propre à la Mésoamérique préhispanique, les cervidés occupent une place particulière, probablement en premier lieu par le fait qu'il s'agit des animaux les plus grands en taille parmi la faune comestible disponible. Jamais véritablement domestiqués dans une économie où l'élevage était, de fait, limité à seulement deux espèces à viande de plus petite taille, ils ont constitué de tout temps la proie la plus recherchée à la chasse. La chasse au cerf a ainsi joué un rôle primordial pour l'apport en protéines des populations, depuis les origines jusqu'à l'époque de la Conquête ${ }^{1}$. Mais les cervidés occupent également une position majeure dans les mythologies et dans les pratiques rituelles des différentes civilisations, ce qui a notamment pu motiver l'instauration de règles particulières pour leur consommation. Si cette place des cervidés dans les aspects rituels est assez bien connue pour la civilisation maya ou chez les Aztèques grâce à l'étude conjointe des textes, des vestiges archéologiques et de l'iconographie, elle semble également importante chez les anciens P'urhépecha du Michoacan, même si elle y demeure nettement moins bien cernée. En effet, bien que le royaume des P'urhépecha, appelés aussi Tarasques par les Espagnols au moment de la Conquête, ait constitué l'une des entités politiques, économiques et militaires majeures dans la Mésoamérique du Xvi ${ }^{\mathrm{e}}$ siècle, le système religieux et l'organisation des croyances de ces derniers restent encore de nos jours très mal connus, car les recherches archéologiques ont été moins nombreuses au Michoacan et la documentation ethnohistorique disponible est plus limitée que pour le Haut Plateau Central. Dans cet article, nous nous efforcerons cependant d'analyser la place du cerf chez les Tarasques grâce à l'étude des informations fournies par différents types de sources, en essayant de déterminer les utilisations des cervidés dans la vie domestique, dans la construction de l'idéologie de l'élite et dans les activités rituelles, ainsi que de montrer en quoi cette figure animale qui représente d'abord, comme ailleurs, la prise de chasse par excellence, joue également un rôle fondamental en relation avec les pratiques guerrières et sacrificielles.

\section{UNE APPROCHE ETHNOHISTORIQUE ET ARCHÉOLOGIQUE}

Pour ce faire, nous nous appuierons très largement sur la Relation du Michoacam. Ce texte, dont le titre originel, Relación de las ceremonias y ritos y población y gobierno de los indios de la provincia de Michoacán [RM], illustre clairement la composition et les thèmes abordés, a été rédigé dans les années 1540 à la demande du vice-roi Antonio de Mendoza. D'un apport considérable par la précocité de sa rédaction, ce document dont l'objectif initial était de décrire les coutumes des 
habitants du Michoacan avant l'arrivée des Espagnols était à l'origine composé de trois sections. Néanmoins, seulement les deux dernières sont aujourd'hui connues, car du premier volet du texte correspondant à la description des rites et des croyances presque tout a disparu. C'est donc sur la deuxième partie, qui reprend le récit mythique de l'arrivée dans la région du lac de Patzcuaro d'un groupe de guerriers nommé uacusecha (Aigles), qui deviendra par la suite le groupe dirigeant du royaume, ainsi que sur la troisième partie, consacrée, elle, à la description de l'organisation du royaume et du déroulement de la Conquête, que notre étude s'appuiera. Il est bien évident que ces deux sections du texte s'inspirent de sources initiales distinctes et poursuivent des objectifs différents. La deuxième partie prétend retranscrire fidèlement le récit, qui était proclamé devant la foule rassemblée lors de certaines fêtes, de l'origine du groupe dirigeant depuis son arrivée dans le Nord du Michoacan jusqu'à l'instauration d'une véritable royauté sous le règne de Tariacuri, vers 1400 apr. J.-C. Elle reprend donc le discours des prêtres, et plus particulièrement du grand prêtre, le Petámuti (Figure 1), qui détenaient une tradition conservée de génération en génération et transmise oralement, puisque les Tarasques ne possédaient pas de système d'écriture. En ce sens, il s'agit d'une source relativement directe, même s'il est indispensable de considérer qu'elle délivre une version de l'histoire voulue par l'élite au pouvoir, et qui est donc aussi orientée en fonction de ses objectifs politiques. De plus, ce message a été dans un second temps retranscrit par un Espagnol qui intervient, pour sa part, dans le cadre de l'implantation coloniale. Ainsi, au-delà de l'interrogation légitime sur la fiabilité historique du récit, le poids de l'intervention du compilateur, particulièrement pour cette deuxième section, est différemment apprécié par les auteurs; en effet, si la narration est qualifiée par certains d'authentiquement tarasque (par exemple, Corona Nuñez 1977 ; Seler 2000 ; Le Clézio 2000), d'autres estiment que son organisation se rapproche nettement de modèles littéraires européens (Pérez Martinez 2000, p. 93 ; Espejel Carvajal 2004, pp. 29, 284). En dépit de ces réserves, une majorité de chercheurs s'accorde à considérer cette deuxième section de la RM comme particulièrement utile pour connaître la conception que l'élite avait de son destin et de son image. La troisième section est, de son côté, plus directement influencée par le contexte historique dans lequel ont été recueillis les épisodes et descriptions évoqués dans le texte. C'est, en effet, principalement sur le témoignage de Don Pedro Cuiniarangari (ou Cuiniharangari) (Figure 1), descendant direct de la famille royale et gouverneur de Tzintzuntzan, que le missionnaire va s'appuyer. Le ton du récit y est nettement distinct puisqu'il s'agit de descriptions concernant l'organisation du royaume sur les plans politique, religieux, militaire, mais également économique et social, suivies d'un récit de l'arrivée des Espagnols au Michoacan. Ces interventions coloniales à plusieurs niveaux de la compilation et de la transcription du texte ne sont pas sans conséquence sur les possibilités d'interprétation des différentes sections du texte, qui doivent s'inscrire dans une démarche critique. 


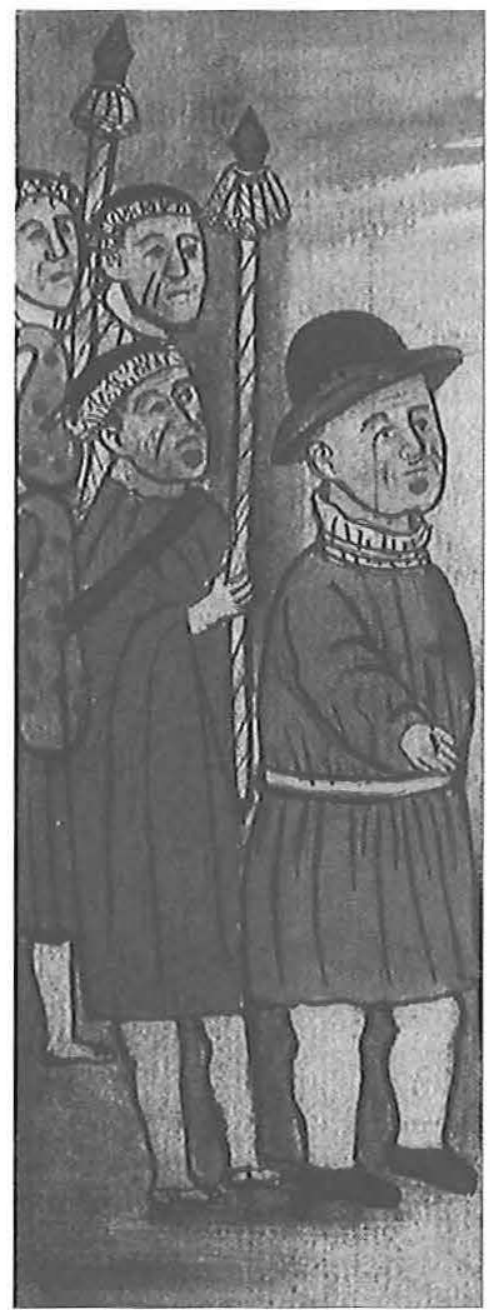

FIG. 1 - Don Pedro Cuiniarangari et le grand prêtre, deux récits pour deux visions de l'histoire du royaume.

C'est ainsi que la figure du cerf occupe dans ces deux parties une place distincte, qui correspond à des niveaux de discours et à des moments de l'histoire du royaume différents. Par ailleurs, le texte est accompagné de planches illustrées qui sont intéressantes aussi à étudier par elles-mêmes, car elles apportent des détails supplémentaires, même si elles sont de facture nettement européenne (Roskamp 2000). Elles seront également utilisées pour compléter notre étude et nous nous attacherons à en décrypter certains messages. 
De façon paradoxale, puisque la section du texte qui leur était spécifiquement consacrée a disparu, c'est peut-être en ce qui concerne les rituels que la RM propose le témoignage le plus authentique. En effet, le compilateur se trouva confronté à un domaine pour lequel il manquait de références dans sa propre culture et qu'il était important de bien évaluer pour l'évangélisation. Les informations concernant le champ des rites et des croyances sont aujourd'hui très largement dispersées dans les deux parties connues du texte et les autres sources ethnohistoriques demeurent de peu d'utilité, car elles n'apportent que de rares précisions sur le sujet ${ }^{2}$. Pour comprendre certains rituels décrits par la RM, ou pour interpréter des détails iconographiques illustrés dans les planches, il est ainsi tentant d'effectuer des comparaisons avec les données, beaucoup plus abondantes, concernant les Mexica. Cette démarche, d'ailleurs adoptée par de nombreux ethnohistoriens et archéologues dans le passé (Seler 2000 ; Corona Nuñez 1946 ; Pollard 1993) peut se justifier par la proximité de ces deux entités politiques majeures du Postclassique et par les parallèles que semblent présenter leurs trajectoires historiques. En effet, comme dans le cas de la civilisation voisine et rivale du Bassin de Mexico, le récit d'une migration originelle transmet la revendication de la part des Tarasques d'une double origine, dans laquelle au moins un groupe de guerriers (les uacusecha) serait arrivé par le nord dans une région lacustre déjà fort peuplée et se serait installé au centre du système de pouvoir par le biais conjoint d'alliances matrimoniales et de conquêtes militaires. Rappelons, à cet égard, que la trop grande proximité avec les traditions aztèques a permis à certains auteurs d'émettre l'hypothèse que le récit tarasque pourrait avoir été contaminé par l'histoire mythique de leurs prestigieux voisins (Michelet 1989). Sans négliger cette interprétation, il faut au moins noter que des parallèles entre les deux traditions peuvent être dressés à de multiples niveaux, notamment concernant l'idéologie et les systèmes religieux (Seler 2000 ; Corona Nuñez 1946, 1977), ces domaines étant peut-être moins facilement manipulables que le simple récit des origines. La RM elle-même précise la proximité des deux civilisations, en soulignant précisément les parallèles idéologiques et religieux : " estos dos reinos eran nombrados, y en estos dos reinos miraban los dioses desde el cielo y el sol » (RM 1977, p. 241).

Outre l'utilité de l'approche comparative, une révision des indices fournis par le matériel archéologique devrait théoriquement permettre de nuancer les informations ethnohistoriques et d'obtenir une vision plus objective et complète de la place du cerf dans la vie quotidienne et les rituels des Tarasques. Malheureusement, face à la richesse des informations apportées par l'étude des textes, il faut reconnaître que les données concernant les cervidés dans les vestiges archéologiques apparaissent comme fortement limitées. Il faut tout d'abord souligner que, bien que les premiers travaux aient été entrepris au Michoacan dès le milieu du siècle passé (voir notamment Acosta 1939; Gali 1946), l'archéologie tarasque n'en est encore qu'à ses débuts : peu de sites ont été fouillés de façon systématique 
et les données même issues de ces quelques fouilles ne sont pas publiées de façon exhaustive. D'ailleurs, quand les publications existent, il est parfois bien difficile de replacer les différents objets décrits dans un contexte temporel précis et les matériaux sont ainsi pour la plupart difficilement utilisables. Enfin, les publications qui rendent compte du détail des matériaux trouvés en fouille sont pratiquement inexistantes et, par exemple, les décomptes des ossements de faune, qui devraient servir de base à une étude sur la part des cervidés dans l'alimentation ou dans les activités rituelles, font figure de véritables exceptions et ne sont donc pas représentatives.

Sur le plan de l'iconographie, les données ne sont pas plus abondantes. Au sein des objets qui, stylistiquement ou par leurs caractéristiques techniques, sont indéniablement tarasques, comme certaines sculptures et céramiques, ou encore la métallurgie, les représentations explicites de cervidés sont extrêmement rares. Parmi les sculptures en ronde-bosse, les êtres zoomorphes sont absents en dehors des célèbres coyotes (Williams 1992). En revanche, toujours dans le travail de la pierre, des silhouettes de cervidés ont été retrouvées sur des pétroglyphes, difficiles à dater mais situées dans des secteurs peu éloignés d'établissements postclassiques. La céramique tarasque est, de son côté, décorée principalement de motifs géométriques et si lézards, serpents ou poissons ornent pārfóis les tubes ou les fourneaux des pipes en céramique, aucun cervidé n'a pu être localisé dans. la bibliographie disponible. En revanche, les productions en métal, au sein desquelles les zoomorphes sont fréquents, peuvent fournir quelques exemples utiles pour notre propos. En tout état de cause, les cervidés ne faisaient pas, à l'évidence, partie des représentations les plus fréquentes chez les Tarasques et, par conséquent, les données que nous traiterons ici ne constituent qu'une synthèse provisoire.

En dépit de ce constat, le recoupement des informations recueillies devrait permettre de dégager certaines hypothèses sur la place occupée par les cervidés dans l'idéologie des Tarasques. Nous verrons que les mentions réitérées de ces animaux dans la RM et les quelques informations archéologiques enregistrées permettent de distinguer une double influence dans les mythes et les rituels, certains s'inscrivant clairement dans un système de codes rituels très largement partagés à l'échelle mésoaméricaine, alors que d'autres semblent se référer à des apports plus spécifiques du Nord du Mexique et caractéristiques du Postclassique. Les liens qui unissent le cerf à la guerre et aux pratiques sacrificielles constituent un système d'associations symboliques clairement identifiable et sera analysé dans un premier temps. Nous essayerons de décrypter ensuite les moyens utilisés par l'élite dans sa recherche d'affirmation politique et identitaire, en examinant notamment le rôle qu'elle fait jouer aux cervidés dans l'idéologie. 


\section{Chasse, guerre, SACRIFICE}

La lecture de la RM, comme les informations issues de l'analyse des vestiges archéologiques, met en évidence un ensemble de fonctions symboliques qui placent le cerf au centre de pratiques sacrificielles. De proie de chasse, le cerf devient ainsi une victime sacrifiée devant un temple et offerte aux divinités.

\section{Le cerf, une proie privilégiée}

Comme dans beaucoup d'autres régions de Mésoamérique, le cerf apparaît avant tout chez les P'urhépecha comme la proie par excellence.

De nombreuses mentions de chasses au cerf figurent, de fait, dans la deuxième partie de la RM : ainsi, les dirigeants du groupe uacusecha, comme le premier d'entre eux au temps de la migration, Ticatame, ou les neveux du roi Tariacuri, Hiripan et Tangaxoan au moment des guerres d'annexion précédant la création du royaume, passent beaucoup de temps dans les bois à chasser le cerf. Cette activité affichée par les personnages placés à la tête de la hiérarchie sociale et son association étroite avec la récolte de bois destinée à alimenter les bûchers sacrés (Faugère K. 1998) contribuent à donner à la chasse une connotation symbolique et renvoient à des activités rituelles liées au statut de guerrier. En dehors de la guerre, ces protagonistes semblent en effet être occupés à des tâches rituelles bien spécifiques ; ils s'isolent dans les bois, se recueillent dans les grottes pour fumer la pipe et "s'imprégner 》 de fumée, s'épuisent à porter du bois pour les bûchers sacrés et à chasser inlassablement le cerf.

Cette position privilégiée des cervidés est également perceptible dans la hiérarchie des serviteurs du roi : le quanicoti est le majordome général des chasseurs, mais il est chargé d'une fonction particulière, celle d'apporter les lièvres et les cerfs au cazonci, les autres proies devant être livrées à part. Cette fonction de proie de chasse privilégiée est d'ailleurs clairement illustrée dans la planche XXVIII qui représente les différentes corporations regroupées autour du dirigeant. Chaque groupe d'artisans y est représenté par des personnages assis, accompagnés d'outils ou de productions permettant de les identifier : le majordome des agriculteurs (tareta uaxatati) brandit un plant de maïs, les charpentiers (tecacha) une hache, les plumassiers (uzquarecucha) sont assis à côté d'un faisceau de plumes, les pêcheurs (uarucha) à côté d'un filet à manche et de poissons. Les chasseurs (quanicocha), quant à eux, apparaissent dans l'angle inférieur de la planche tenant un arc de grande taille et des flèches, tandis que, devant eux, gît un cerf, les quatre pattes attachées par une corde (Figure 2). L'espèce ici représentée est assez clairement identifiable : on reconnaît le museau allongé, des andouillers courts recourbés vers l'avant, les membres minces et la queue assez longue du cerf à queue blanche, Odocoileus virginianus. Ce cervidé 


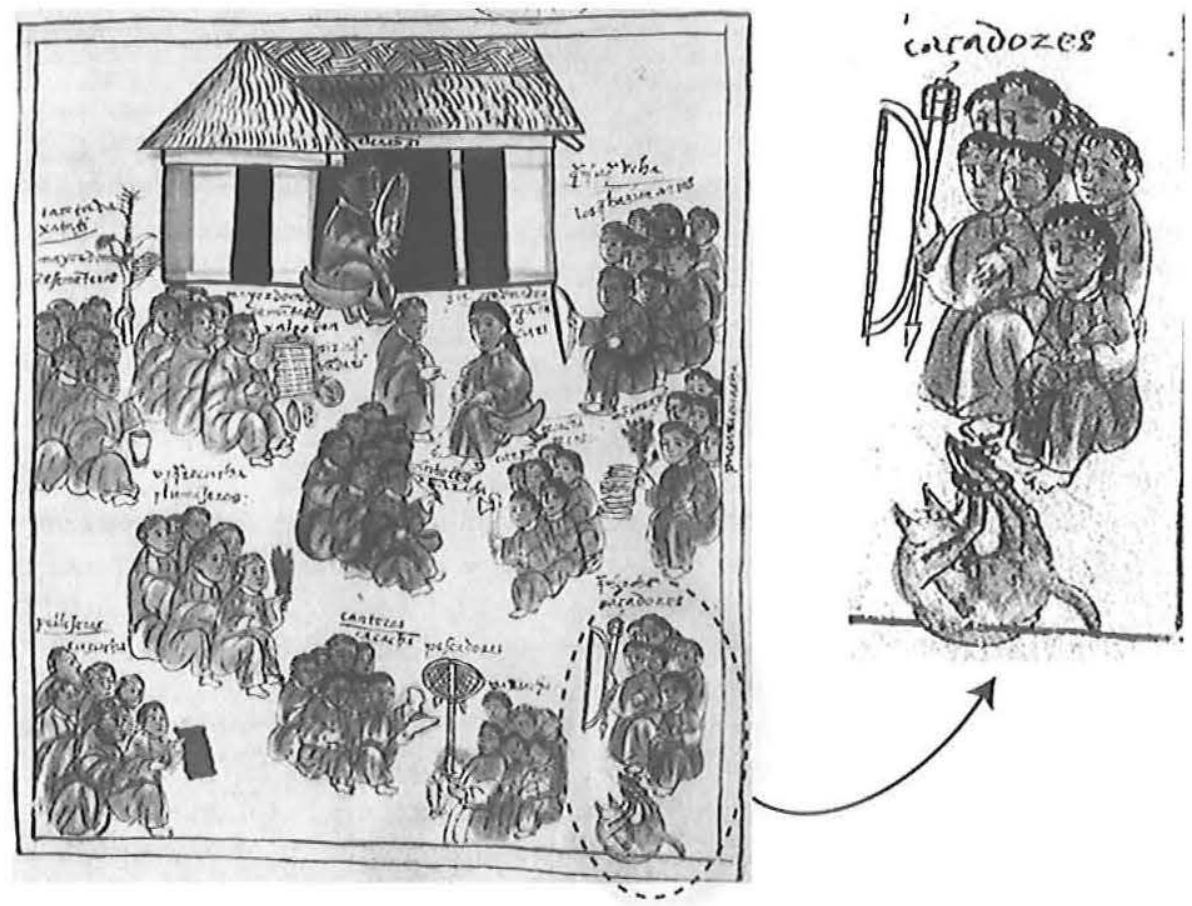

FIG. 2 - Le Cazonci devant quelques corporations et détail du groupe des chasseurs (d'après la planche XXVIII, in RM 1977).

constitue l'espèce présente au Michoacan à l'époque préhispanique et était encore très abondant dans la région il y a une cinquantaine d'années.

Sa taille fournit d'abord au chasseur une quantité appréciable de viande, mais sa peau, ses os et ses andouillers représentent également des matières premières appréciées, susceptibles d'être transformées en produits (couvertures, vêtements...) et en outils (poinçons, retouchoirs...).

Malheureusement les vestiges archéologiques n'apportent que peu d'informations complémentaires. La consommation de viande de cerf dans le cadre de la vie domestique pourrait être en principe évaluée en étudiant les proportions relatives des espèces consommées retrouvées dans les déchets alimentaires. Si l'on s'en tient aux seules données concernant le Postclassique récent, l'unique référence disponible à ce jour est la thèse de doctorat de Migeon (1990, pp. 357, 358) qui a travaillé sur les sites tarasques proches de Zacapu. Ces établissements seraient la manifestation de l'installation de groupes tarasques dans la région et correspondraient au moment où, dans le récit de la deuxième partie de la RM, 
Ticatame et ses uacusecha pénètrent au Michoacan, c'est-à-dire entre 1200 et 1300 approximativement (Michelet 1998 ; Espejel Carvajal 2004, p. 95). Parmi les espèces identifiées dans les déchets domestiques localisés dans plusieurs unités d'habitation du site de Las Milpillas (MICH.95), le cerf n'occupe qu'une faible place (en moyenne $5 \%$ ), derrière les oiseaux (divers, canards et perdrix : en moyenne $33 \%$ ) et les rongeurs (en moyenne $57 \%$ ). Les données issues de simples sondages stratigraphiques réalisés sur le site d'El Palacio (MICH.23), site tarasque important qui dominait l'ancien lac de Zacapu, montrent des proportions assez comparables, sauf que les restes de tortues d'eau douce sont là particulièrement abondants (Migeon ibid., p. 187). Ces données quantitatives semblent montrer que les cervidés n'étaient apparemment pas les animaux les plus consommés quotidiennement et que les populations tarasques exploitaient d'abord leur milieu proche pour se fournir en viande. Il aurait été évidemment très intéressant de pouvoir évaluer et comparer les proportions des différents types de viandes consommées lors des fêtes et d'essayer d'entrevoir si certains interdits alimentaires pouvaient exister, ce qui est malheureusement hors de notre portée.

Par ailleurs, la taille des os longs de cervidés permettait de les utiliser pour fabriquer différents types d'objets. Ces os transformés en outils se retrouvent d'ailleurs dans l'ensemble de la Mésoamérique, notamment des poinçons fréquemment réalisés sur les métapodes et des aiguilles, que l'on retrouve à toutes les époques. Au Michoacan, de nombreux exemples de ces outils sont connus et, même s'ils manquent dans leur majorité de contextes de provenance précis, il est possible d'admettre que certains sont de fabrication tarasque. En revanche, la présence d'andouillers ou d'os du crâne n'est pratiquement jamais mentionnée, peut-être parce que les zones artisanales demeurent peu fouillées ou à cause de certaines pratiques de découpe.

$\mathrm{Si}$, en l'état actuel des connaissances, la chasse au cerf n'apparaît donc pas comme une activité primordiale pour assurer la vie quotidienne des populations, quelques rares pétroglyphes figuratifs retrouvés dans la même région du malpais de Zacapu font indéniablement référence à la chasse de quadrupèdes dont la taille permet de supposer qu'il s'agit de cervidés (Figure 3a). L'étude des représentations rupestres de la région a montré que les motifs gravés semblent avoir été réalisés dans des lieux précis et qu'ils auraient plutôt eu des fonctions rituelles (Faugère K. 1997). En ce sens, les scènes de chasse gravées à proximité des sites tarasques ne se référeraient pas forcément à la représentation de chasses ordinaires, mais à des chasses qui se déroulaient dans un cadre rituel. Par ailleurs, il faut noter que ces scènes sont associées spatialement à des blocs où sont figurés des combats ou des guerres : des anthropomorphes semblent s'affronter avec des armes et des individus sont transpercés de part en part par des lances (Figure 3b). Il est ainsi possible de supposer que certains de ces pétroglyphes figuratifs représentaient des chasses rituelles et qu'ils renvoyaient, par ailleurs, à l'association chasse-guerre qui se révèle à la lecture de la deuxième section de la RM. 


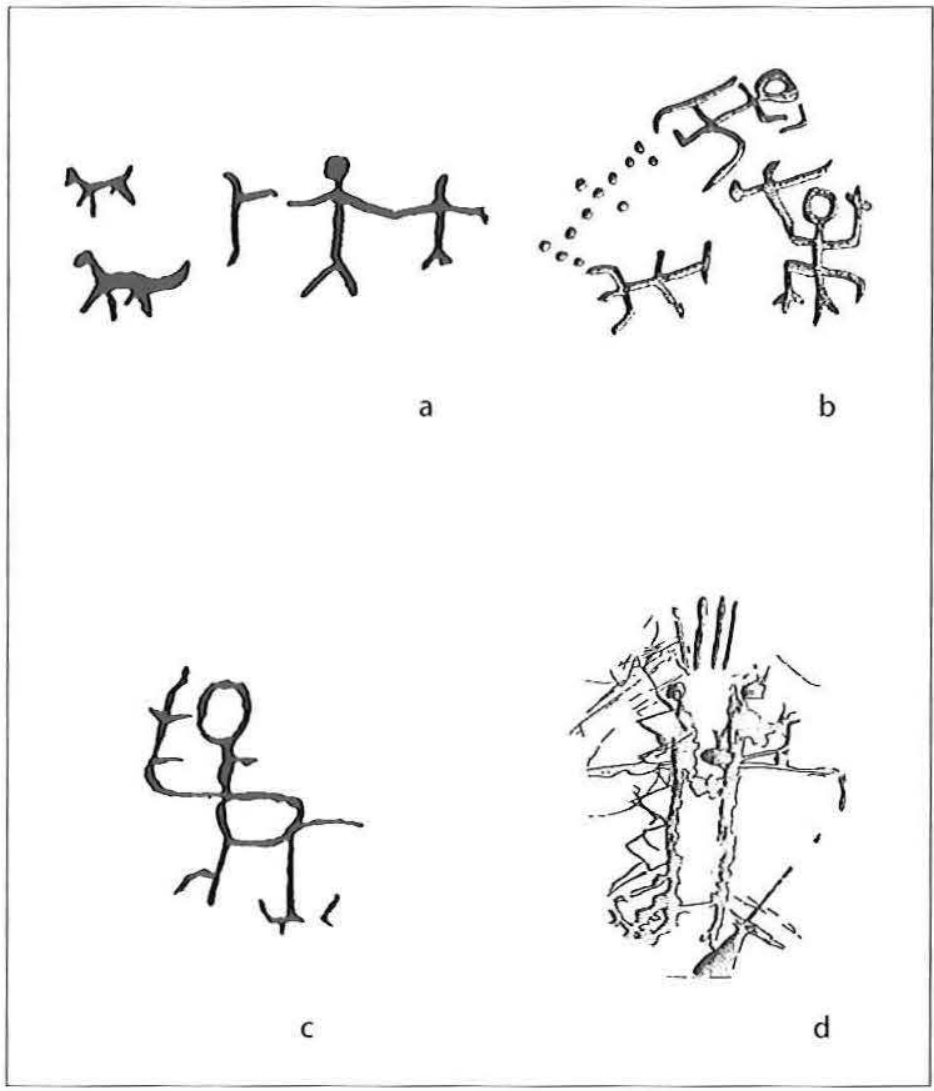

FIG. 3 - Pétroglyphes. Représentations de scènes de chasse et de combats, de guerriers et d'un possible cervidé (d'après Faugère K. 1997).

\section{De l'animal chassé à l'animal sacrifié}

Si les « seigneurs bûcherons » passent beaucoup de temps dans la forêt, c'est, selon la deuxième partie de la Relation du Michoacan, d'abord pour nourrir les dieux avant de nourrir les hommes. En effet, la chair de cerf aurait constitué, au même titre que l'offrande de fumée, un aliment destiné aux divinités « al sol y a los dioses celestes, y a las cuatro partes del mundo, y a la madre Cuerauáperi 》 (RM 1977, p. 17).

Cette fonction d'offrande privilégiée se retrouve à plusieurs reprises dans le texte de la RM. Lors de l'intronisation du cazonci, alors qu'il devient le représentant sur terre de Curicaueri, lui sont offerts des présents dont la valeur symboli- 
que est indéniable : des arcs et des flèches, des cerfs, des fruits, du vin, des tamales de maïs et des metates (RM 1977, p. 226). On retrouve cette notion d'offrande destinée aux hommes incarnant une divinité sous une autre forme dans la troisième partie du texte : en effet, le premier cadeau que le cazonci fait aux soldats espagnols à leur arrivée au Michoacan est composé de cinq cerfs ${ }^{3}$ capturés apparemment lors d'une chasse rituelle, ainsi que d'autres biens précieux comme des disques d'or et des coiffes.

Peu de détails nous sont parvenus pour connaître la forme exacte que revêtait l'offrande. Néanmoins, un paragraphe de la Relation indique sans équivoque que, si les cerfs étaient «fléchés » et étaient donc probablement morts au moment de l'offrande, ils pouvaient aussi être capturés vivants afin d'être sacrifiés devant un temple.

Tomaron alli un venado, y tomó toda la gente mucha rama y leña, que iban en dos procesiones, $y$ llegaron asi al pueblo y llevaban el venado delante, y hicieron um gran fuego que se alzó una gran llama y humo cabe la trox del dios Urendequaúecara de Curínguaro, y sacrificaron aquel venado al pie de la trox y atáronle y pusiéronle a las espaldas. (RM 1977, p. 66)

D'autres passages de la Relation permettent de mieux comprendre l'articulation entre chasse et sacrifice, notamment en relation avec les activités guerrières.

\section{Une homologie homme-cerf}

Les liens qui unissent l'homme et le cerf dans le cadre des pratiques sacrificielles peuvent être mis en évidence à travers la partie de la Relation de Michoacan décrivant le traitement que le roi Tariacuri réserve au prêtre Naca. Ce récit est assez largement développé puisqu'il occupe plusieurs sections du texte et est illustré par pas moins de trois planches (planches IV à VI).

Cet épisode se déroule dans le contexte où le dirigeant du groupe uacusecha tente d'imposer sa domination sur la région du lac de Patzcuaro. Il se heurte alors à la résistance et à l'hostilité des populations de pêcheurs des îles. Les « Chichimèques » assiègent les gens des îles, contraints, comme le montre la planche IV, à rester reclus, à ne plus pouvoir s'approvisionner et rejoindre leurs champs. Naca est chargé par un groupe d'opposants d'aller lever en secret des troupes afin de mettre fin aux attaques [ou pressions] des Chichimèques et il est envoyé par le seigneur Zurumban à Curínguaro, afin de sceller une alliance (" los chichimecas son poca gente y juntándonos los destruiremos », RM 1997, p. 48).

Tariacuri, averti par un complice, imagine alors un stratagème pour éliminer le prêtre et il charge ses frères, Zétaco et Aramen, de le mettre en œuvre. Il s'agit d'abord d'organiser une chasse fictive au cerf afin de capturer le prêtre dissident. La mise en scène est illustrée par la planche V (Figure 4). On y voit le bord de la lagune avec la barque qu'a laissée Naca. Les espions surveillant son arrivée sont 


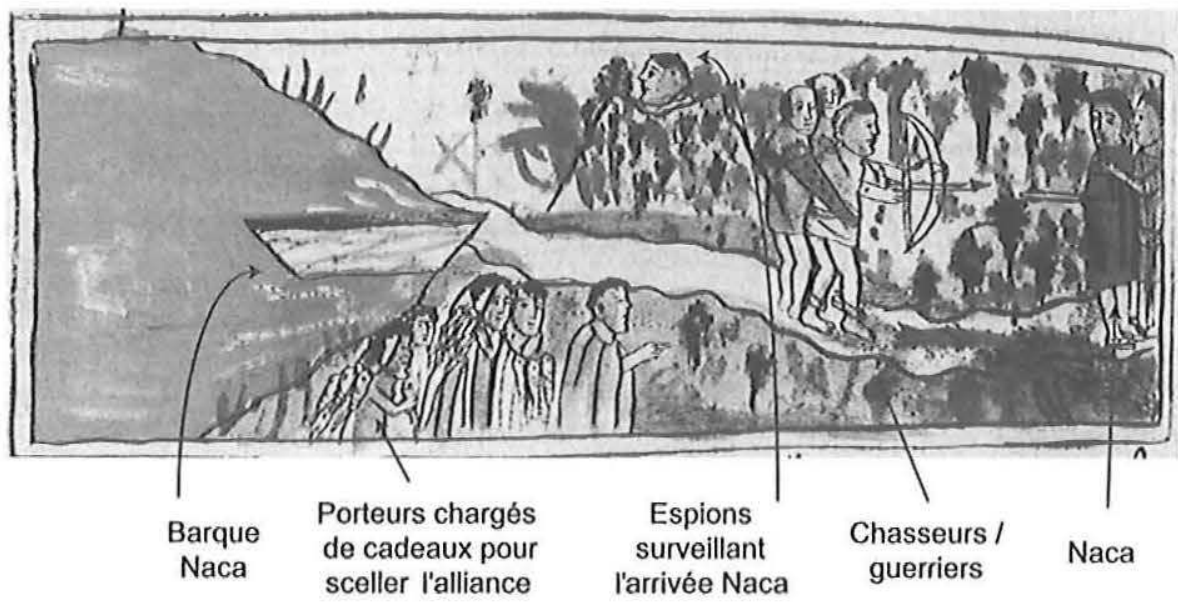

FIG. 4 - L'épisode de Naca, l'embuscade (d'après la planche V, in RM 2000).

postés en haut du mont Harazindo. Un groupe de porteurs est chargé de poissons qui doivent servir de cadeaux pour sceller les alliances entre les opposants. Mais les deux frères, vêtus en quanguariecha (ou quengariecha, c'est-à-dire " hommes vaillants ») et portant la tonsure caractéristique de l'ordre guerrier, se trouvent sur le chemin (Figure 5). Ils ont revêtu le vêtement court brun, les bandes de cuir ornées de sabots de cerf sont attachées à leurs chevilles et leur carquois de peau de jaguar confirme leur proximité familiale avec le cazonci. L'un d'eux est en train de bander son arc, mais une flèche est déjà fichée dans la poitrine de Naca qui lui fait face. Ce dernier porte un vêtement long en textile, un labret et une guirlande de fils sur la tête, coiffe caractéristique des prêtres. Le sol proche de Naca porte des traces de couleur rouge. Pour trouver une explication à la présence de cette couleur sur le sol, il faut se reporter au texte et aux recommandations faites par Tariacuri à ses frères. Ils se sont scarifiés les oreilles et, avec le sang, ont imité sur le sol des empreintes de pattes de cerf afin de faire croire à Naca qu'ils en avaient blessé un et qu'ils étaient à sa recherche. Naca, blessé et surpris, essaie de convaincre ses assaillants de sa bonne foi, mais rien n'y fait : il est conduit à Tariacuri qui ordonne qu'il soit sacrifié. Mais la vengeance se poursuit : le corps de Naca est découpé en morceaux.

Tomad a Naca [...] que le cuezan los dos muslos, que los lleven a Zurumban que le envió a hacer gente. Que haga con ellos la salva a los dioses y el cuerpo y las costillas llévenlo a los isleños para que hagan la salva, y los dos brazos llévenlos a Curínguaro, para hacer la salva. (RM 1977, p. 56)

La planche VI accompagne ce texte (Figure 6) : on y voit à gauche la préparation du banquet avec des pièces de corps humain émergeant d'une marmite et, 


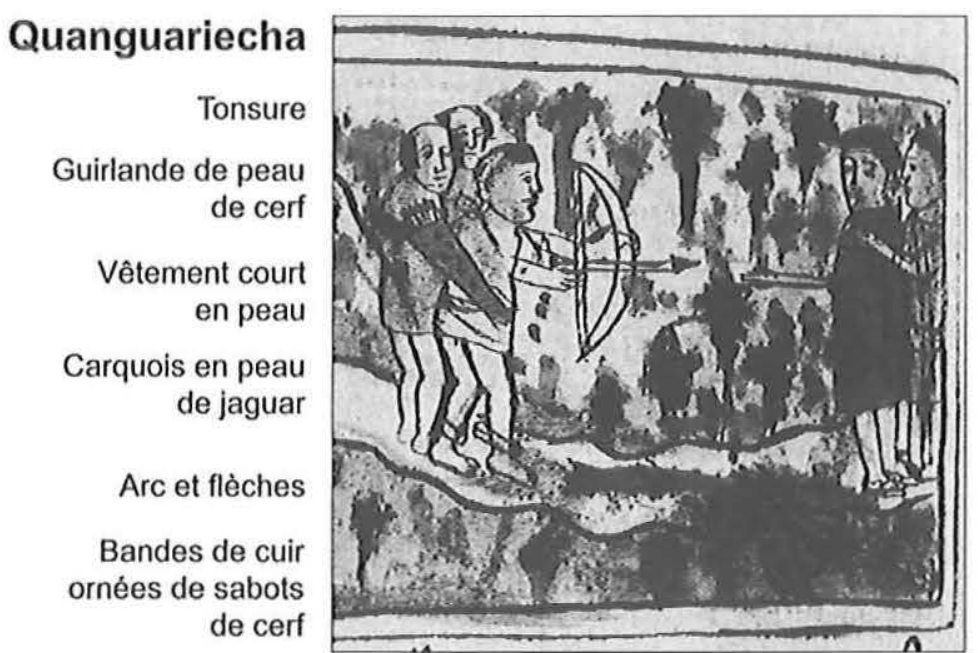

FIG. 5 - Détail de la planche V (RM 2000).

\section{Naca}

Guirlande

de fils

Labret

Vêtement

long en

textile

Le sang de l'autosacrifice remplace le sang de cerf

à droite, Zurumban en train de manger les mains, tandis que des femmes tentent de vomir au bas de la planche lorsqu'elles apprennent ce qu'elles viennent d'ingérer.

Les relations qui unissent la guerre, la chasse et le sacrifice sont ainsi particulièrement bien illustrées dans ce passage : la guerre prend la forme d'une chasse au cerf, elle est aussi un moyen pour alimenter les dieux. Le passage concernant l'épanchement de sang réalisé par les guerriers pour capturer Naca est également intéressant et, afin de l'interpréter correctement, il convient de revenir sur la manière dont les anciens P'urhépecha pratiquaient l'autosacrifice. Dans la RM, toutes les mentions d'autosacrifices se réfèrent aux oreilles; les «hommes vaillants » et les seigneurs devaient offrir leur sang en s'entaillant profondément les oreilles (Terán Elizondo 2000 ; Pollard 1993). L'autosacrifice des oreilles était notamment pratiqué lors de la fête Purecotaquaro, au cours de laquelle se déroulait également le sacrifice des délinquants (Espejel Carvajal 2004, p. 161). Le sang est une offrande divine, tant celui produit sur le champ de bataille que celui de l'autosacrifice, qui se réalisait principalement avec des lames d'obsidienne (Darras 1998).

Y tenían lo por mal descalabrarse, y en descalabrándose alguno, alimpiabase con la mano la sangre, porque no cayese en el suelo, y ruciabanla con los dedos hacia el cielo, para dar de comer a los dioses. (RM 1977, p. 36)

Dans l'épisode étudié ici, le sang, qui est répandu à la suite d'un autosacrifice des guerriers, est destiné à simuler celui d'un cerf blessé. Le sang de l'auto- 


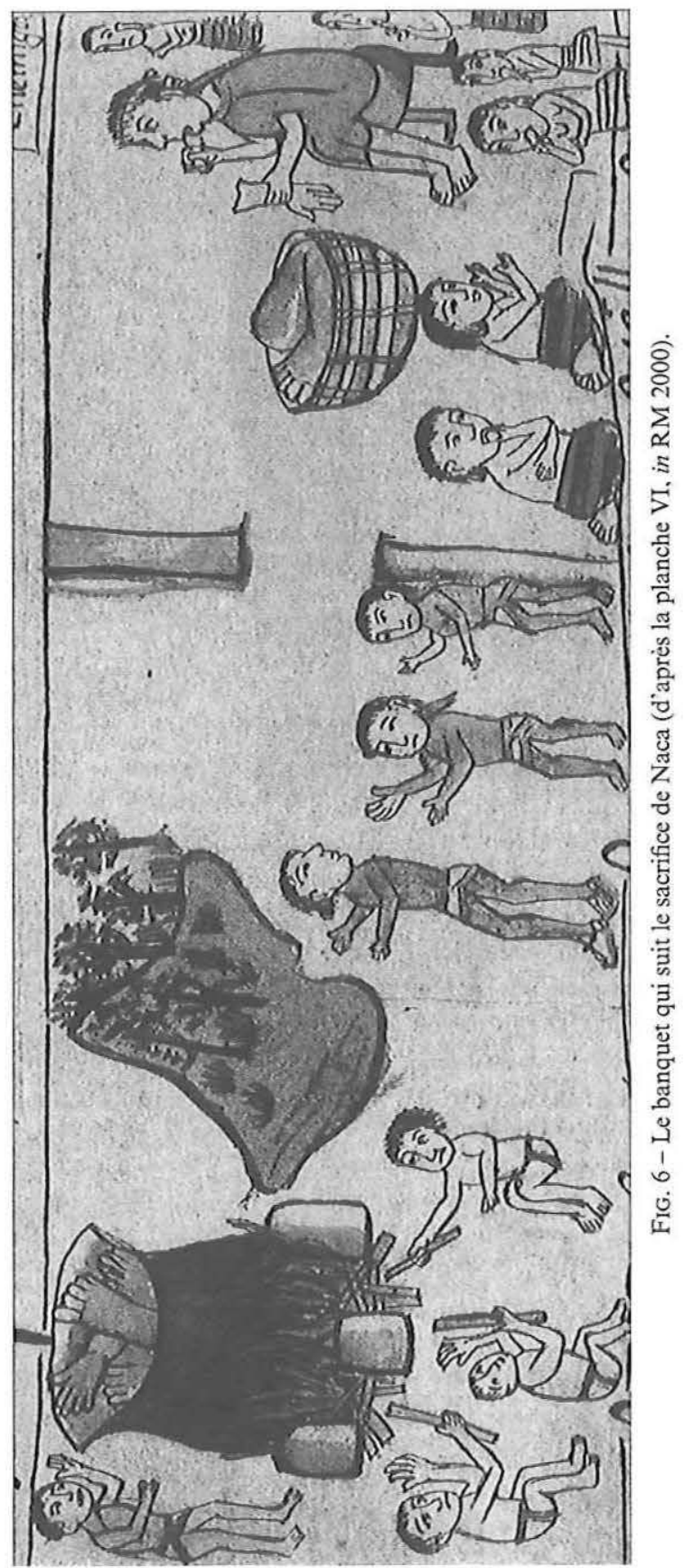


sacrifice remplace le sang du cerf, la chair humaine est un aliment au même titre que la chair de l'animal. Une homologie entre le cerf et l'homme se dessine, là aussi, clairement.

Quels sont les vestiges archéologiques qui pourraient aller dans le sens de cette homologie homme-cerf? et comment la fonction sacrificielle des cervidés pourrait-elle être repérable ? Pour répondre à la première question, il est bien évident que les caractéristiques de la faune de la Mésoamérique préhispanique plaçaient les cervidés dans une position particulière. En effet, il s'agissait des seuls animaux, en dehors de l'homme, dont les os longs pouvaient servir de support à la fabrication d'outils ou d'objets rituels de grande taille. Dans ce contexte se pose précisément à l'archéologue le problème de l'identification de l'espèce, car les ossements retrouvés en contexte archéologique sont le plus souvent fragmentés. Ainsi, des analyses spécifiques peuvent se révéler nécessaires pour déterminer si l'os est d'origine humaine ou animale et, dans ce dernier cas, pour en déterminer l'espèce. Outre l'élaboration d'outils destinés à des usages domestiques, certains artefacts en os de cervidés sont considérés, en Mésoamérique, comme des instruments destinés aux pratiques d'autosacrifices, en particulier dans la zone maya (Pohl 1981). Au Michoacan, des objets en os localisés dans des sépultures du Classique récent font partie des tombes les plus riches qui seraient celles de guerriers (Pereira 2007). Mais on sait aussi que, toujours à l'époque classique, certains exemplaires en os de jaguar décorés d'une iconographie clairement Teotihuacan étaient présents dans cette même région (Pereira, communication personnelle). Malheureusement les données concernant la période suivante sont moins abondantes. Pour le Postclassique, peut-être plus que pour les époques précédentes, le problème majeur réside dans la confusion possible entre l'os humain et l'os de cervidé, car certains objets rituels auraient pu être fabriqués indifféremment dans l'une ou l'autre matière première. C'est en particulier le cas des omichicahuaztli, ces racles très fréquentes à l'époque tarasque qui étaient utilisées comme instruments de musique lors des cérémonies funèbres, en particulier lors des funérailles des guerriers morts au combat (Pereira 2005). Si l'essentiel de ces racles était élaboré sur des os humains, un probable fragment d'omichicahuaztli sur un os de cervidé provient néanmoins d'une tombe d'enfant localisée sur le site de San Antonio Carupo (Faugère K. 1996), où il était associé à un grelot de cuivre. L'échantillon disponible est trop limité pour que nous puissions interpréter la substitution de l'os humain par l'os de cervidé, même si cette piste demeure intéressante.

En ce qui concerne le rôle sacrificiel des cervidés, les échantillons de faune disponibles sont, comme cela a déjà été dit, trop insuffisants. Néanmoins, nous avons localisé parmi les productions indéniablement tarasques des objets en forme de têtes de cervidés qui pourraient renvoyer à un contexte sacrificiel (Figure 7). Ces petits masques faisaient partie d'un ensemble de grelots et de pendentifs, probablement réalisés dans un alliage à base de cuivre ${ }^{4}$. Si les grelots 

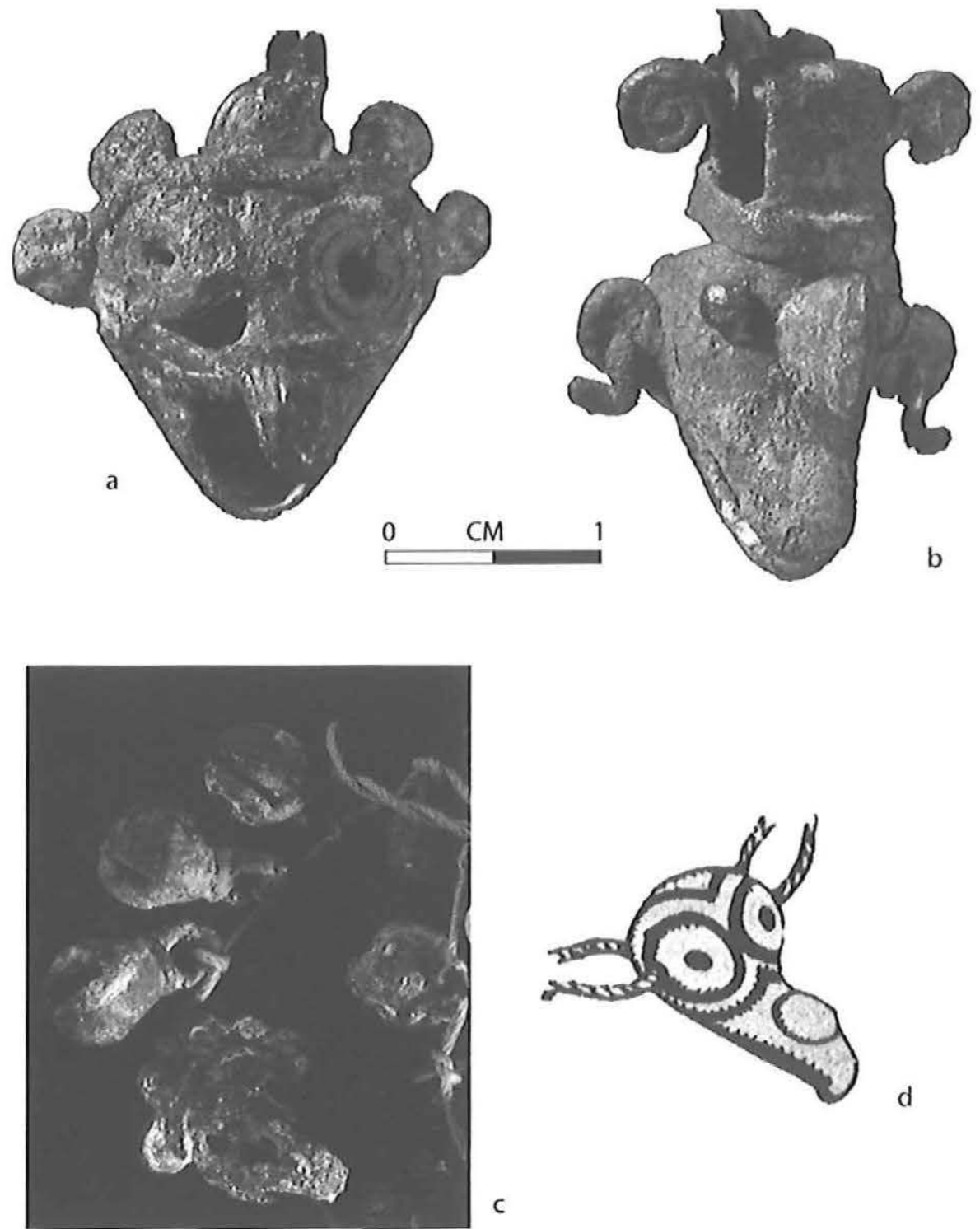

d

FIG. 7 - Métallurgie : (a) masques en forme de têtes de rapace (collection particulière) ; (b) masques en forme de tête de cervidé (collection particulière) ; (c) grelots associés (collection particulière) ; (d) une tête d'aigle (?) en os (d'après Corona Nuñez 1946). 
sont simples et peu décorés, les masques sont plus intéressants sur le plan iconographique. En effet, plusieurs espèces animales sont représentées : outre le cervidé, on trouve un autre mammifère au museau marqué, mais également un oiseau au long bec crochu ouvert, avec des yeux circulaires perforés en leur centre (Figure 7). Même si la fonction précise de cette série de pièces est méconnue, on sait que les objets en métal étaient considérés comme des biens précieux; ils constituaient des éléments de parure et des insignes de prestige pour les nobles et avaient également une connotation religieuse. Ils pouvaient notamment être portés par les victimes lors des sacrifices (RM 1977, p. 158) et faisaient souvent partie des offrandes déposées dans les sépultures des membres de l'élite dirigeante. Le fait que soit représentée une tête de cervidé précisément dans cette matière première, alors que cet animal est, par ailleurs, pratiquement absent des registres iconographiques tarasques, renvoie peut-être à sa fonction symbolique.

\section{LE CERF MYTHIQUE DE CUPANZIEERI}

Une lecture de la troisième partie de la Relation de Michoacan permet de relever un aspect sensiblement différent attribué aux cervidés par les Tarasques, en lien avec un être mythique appelé Tuitze (Seler 2000 ; Corona Nuñez 1946) ou Tuycen (Terán Elizondo 2000). Celui-ci est décrit comme un cerf dont la nuque est ornée de crins et qui possède également une longue queue. Dans la mythologie tarasque, ce cerf appartient à la sphère de "los dioses primogénitos » ou de " la mano derecha », c'est-à-dire du nord, le lieu de résidence des ancêtres, mais aussi celui des âmes des guerriers sacrifiés, qui s'identifient aussi avec les premiers hommes. C'est pourquoi Seler (2000) estime que cet être mythique se réfère également aux origines du genre humain. Par ailleurs, le nord est également considéré comme étant la place du jeu de balle, ce qui nous renvoie à l'origine de ce cerf mythique.

En effet, pour comprendre le rôle de ce grand cerf dans la cosmogonie p'urhépecha, il faut évoquer le mythe de Cupanzieeri ou Apantzieeri, « le joueur de pelote ", raconté dans la RM, et de la partie de jeu de balle qui va aboutir à sa mort par sacrifice. Cupanzieeri affronte une autre divinité, Achuri Hirepe, au jeu de balle, est vaincu, puis sacrifié à Jacona (« maison du soir »), lieu d'origine du vainqueur. Devenu adulte, son fils posthume, Sirahtatahperi (« du lignage de son père »), qui ne serait autre que Curicaueri dans son aspect de dieu du soleil levant (Corona Nuñez 1946, p. 17), se rend à la chasse. Il rencontre alors un iguane qui lui annonce que celui qui l'a élevé n'est pas son vrai père qui, lui, a été sacrifié. Après l'avoir vengé, il déterre la dépouille de son père et la prend sur ses épaules pour la rapporter. Mais, sur le chemin du retour, il passe par un champ où abondent les perdrix et, afin de les tirer, il pose la dépouille de son père à terre. C'est alors que ce dernier reprend vie et se transforme en cerf qui a, cependant, la 
particularité de posséder des crins sur la nuque, élément qui constituerait, selon Corona Nuñez (1946), l'un des attributs du dieu solaire. Une étude de l'étymologie des noms des principaux protagonistes permet de mieux appréhender le sens de ce mythe. Si Seler (2000) avoue ne pas savoir interpréter le nom de Cupanzieeri, Corona Nuñez (idid., p. 17) le traduit par "soleil pris par les cheveux " ou " dépouillé de ses cheveux », le soleil couchant, ce qui nous renvoie également à une symbolique de la chevelure associée à la guerre, connue notamment pour les Mexica (López Austin 1989). Le nom Achuri Hire ne signifie rien d'autre, selon le même auteur; que "la nuit qui se presse" ou encore "les innombrables de la nuit », c'est-à-dire les étoiles, ou, selon Seler (2000), « le dominateur, la nuit ».

Une autre version de ce mythe d'origine est mentionnée par Pollard (1993), citant Hurtado Mendoza (1986) : c'est le dieu soleil Curicaueri qui se crée lui-même une compagne, Achuri Hirepe, qui serait une divinité lunaire. Cette dernière, enceinte, tue Curicaueri et l'enterre à l'est. La même nuit, elle met au monde un fils, Sirahtatahperi. Ce fils lui-même engendre Tuitze qui est envoyé sur terre sous la forme d'un cerf afin de vivre parmi les humains.

Ainsi, le cervidé occupe dans ce mythe un rôle important où il incarnerait un aspect du soleil après que celui-ci eut vaincu les forces de la nuit. Ces noms de divinités correspondent, en effet, aux différents aspects liés aux positions du dieu solaire entre voûte céleste diurne et voûte nocturne, entre deux positions principales opposées : au zénith, Curicaueri et, au nadir, Achuri Hirepe.

On sait enfin que, lors de la fête de Cuingo, on préparait des pains de végétaux en forme de cerfs auxquels on donnait le nom de tuitze. La fête de Cuingo correspond à la Tlacaxipeualiztli mexica, c'est-à-dire qu'elle correspond à la fête de rajeunissement du soleil, celle du renouvellement de la croissance végétale et du début d'un cycle calendaire. La métamorphose du héros en tuitze le relie donc à cette fète. Le fait que de la nourriture ait été préparée en forme de cerf indique que le dieu était symboliquement offert et consommé lors de la cérémonie, selon une tradition qui semble commune avec les Mexica.

Une représentation de ce cerf mythique pourrait faire partie des corpus iconographiques des roches gravées des abords des sites Las Milpillas (MICH.95) et El Palacio (MICH.23). Outre les scènes de chasse et de combat déjà mentionnées, une gravure isolée pourrait, en effet, le représenter (Figure 3c). Bien que s'agissant de toute évidence d'un cervidé, l'animal possède des caractéristiques particulières dont une longue queue, des appendices sur le cou, dont certains pourraient représenter les crins, tandis que ses membres inférieurs sont ramifiés, comme souvent les membres inférieurs des anthropomorphes représentés dans les pétroglyphes. Ces particularités laissent penser qu'il pourrait s'agir d'une représentation d'un animal mythique, le Tuitze, et non d'une figure naturaliste.

Pour terminer, le mythe de Cupanzieeri permet aussi de mesurer et de mieux comprendre l'impact que la vision des chevaux exerça dans l'esprit des derniers Tarasques à l'arrivée des Espagnols. En effet, par leur taille et par leur crinière, ils 
pouvaient être facilement confondus avec le cerf mythique de la légende de Cupanzieeri. La RM retransmet clairement cette confusion : " [...] que el cielo se junta con el mar; y de allí debian de salir pues aquellos venados que dicen que traen » (RM 1977, p. 241).

Le cerf paraît ainsi représenter une entité associée au soleil, mais aussi un ancêtre divin des hommes. La partie de jeu de balle fait référence, comme dans de nombreux cas en Mésoamérique, à la lutte des forces de la lumière et des ténèbres au moment où le soleil disparaît à l'horizon et ressurgit à l'orient. Par son appartenance à la sphère des " dioses primogénitos », il est également associé à la guerre et au sacrifice. Les cerfs interviennent ainsi à différents niveaux de la pensée et des rituels comme une entité complexe qui se réfère néanmoins indéniablement au soleil, aux ancêtres, à la guerre et au sacrifice. Par ailleurs, il est particulièrement frappant de voir, dans la RM, comment cette valeur symbolique, riche et complexe, est utilisée et, peut-être aussi, exploitée par l'élite au pouvoir, détentrice de la tradition orale.

\section{Le Cerf, Curicaueri et L'ÉLITE GUERrière}

Après avoir examiné la place qui est faite au cerf dans les rituels et les croyances, nous souhaiterions examiner plus en détail l'utilisation de cet animal par l'élite qui était au pouvoir au moment de la Conquête, les uacusecha, en nous appuyant donc sur la deuxième section de la RM. Comme cela a déjà été signalé, la chasse au cerf fait partie des activités principales des héros qui sont à l'origine de la création du royaume et, en ce sens, doit être vue comme un élément identitaire de l'appartenance au groupe dominant, même s'il est bien évident que ce discours renvoie aussi directement à la dimension guerrière et sacrificielle associée à ces animaux. Sous forme de cuir tanné ou, au contraire, dont on avait conservé les poils, la peau sert pour la fabrication de couvertures et elle est aussi utilisée pour composer différentes pièces des vêtements distinctifs de l'élite guerrière, depuis le costume du cazonci jusqu'aux tuniques des guerriers quanguariecha. Le texte décrit effectivement assez en détail les costumes des différents protagonistes et il convient de revenir aussi sur la représentation des costumes dans les planches qui complètent utilement le texte.

\section{Le cerf et Curicameri}

Dans la deuxième partie de la RM, le cerf est associé au statut des guerriers, les quanguariecha, et du premier d'entre eux, le cazonci. Il est probable que ce choix renvoie à la revendication d'une origine précise par le groupe dirigeant, origine où la chasse et la guerre occupent une place prépondérante, mais le cerf constitue également le lien avec la divinité tutélaire du groupe de migrants qui 
arrive dans la région du lac de Patzcuaro, Curicaueri. Curicaueri est avant tout un dieu guerrier, dieu du feu, mais il est aussi le feu le plus intense de l'univers, c'est-à-dire le soleil (Corona Nuñez 1946 ; Espejel Carvajal 2004). Comme dieu solaire, il peut adopter différentes formes et différents noms, selon sa position dans le ciel. Cette association conjointe avec le soleil et la guerre - comme le fait qu'il représente la divinité tutélaire du groupe des migrants guerriers venus du nord - le rapproche bien évidemment du Huitzilopochtli aztèque, même si certaines comparaisons peuvent également être établies avec Tezcatlipoca. Comme ces divinités, et d'autres encore, Curicaueri est transporté par des porteurs de dieux sous la forme d'un paquet sacré, et le paquet sacré de Curicaueri présente la particularité d'être enveloppé dans une peau de cerf. Ainsi Ticatame affirme à propos des peaux de cerf, " que no es pellejo, ni sirve de pellejo, sino de mantas, porque los curtimos y ablandamos en ellos a muestro dios Curicaueri » (RM 1977, p. 18). On sait que, chez les Mexica, les paquets sacrés, appelés " tlaquimilolli ", pouvaient contenir des plumes, des fragments d'ossements et, parfois même, des fémurs entiers, des pierres précieuses et des miroirs d'obsidienne enveloppés dans des tissus précieux (Olivier 1995). Si le symbolisme du contenu semble assez comparable chez les Tarasques (voir notamment Darras 1998), l'utilisation de la peau comme contenant constitue une particularité du Michoacan, car les Aztèques semblent plutôt avoir utilisé des textiles. Cette coutume d'utiliser des peaux de cerf pour servir d'enveloppe aux paquets sacrés aurait pu être, néanmoins, partagée par d'autres peuples du Nord du Mexique. Par exemple, Di Peso (1974) décrit la découverte d'un probable paquet sacré à Casas Grandes, apparu sous la forme d'un ensemble de restes archéologiques comprenant des fragments de tissu de coton et de peau de cerf, qui aurait pu contenir à l'origine plusieurs objets dont un miroir de pyrite. Ces paquets sacrés faisaient l'objet de véritables cultes et les sources indiquent que l'étoffe ou la peau de l'enveloppe aurait pu correspondre à ce qui constituait, à l'origine, les vêtements du dieu (Olivier 1997, p. 94). Il est ainsi probable que l'utilisation de la peau de cerf pour fabriquer le paquet sacré de Curicaueri indique que les vêtements même du dieu étaient réalisés dans cette matière, d'où l'intérêt d'étudier les costumes du roi, le représentant de Curicaueri sur la terre.

\section{Le cerf et le roi}

La relation établie entre le cerf et le souverain peut être étudiée en se penchant sur la partie du texte décrivant les cérémonies réalisées lors de l'investiture d'un nouveau dirigeant - elles-mêmes illustrées par la planche XL (Figure 8) - et en observant également les différents costumes du souverain sur les illustrations. Il est frappant de constater à quel point les cérémonies décrites chez les P'urhépecha se rapprochent de celles qui se déroulaient chez leurs voisins mexica au moment de l'élection du nouveau Tlatoani. Après la mort du roi, les dignitaires du 


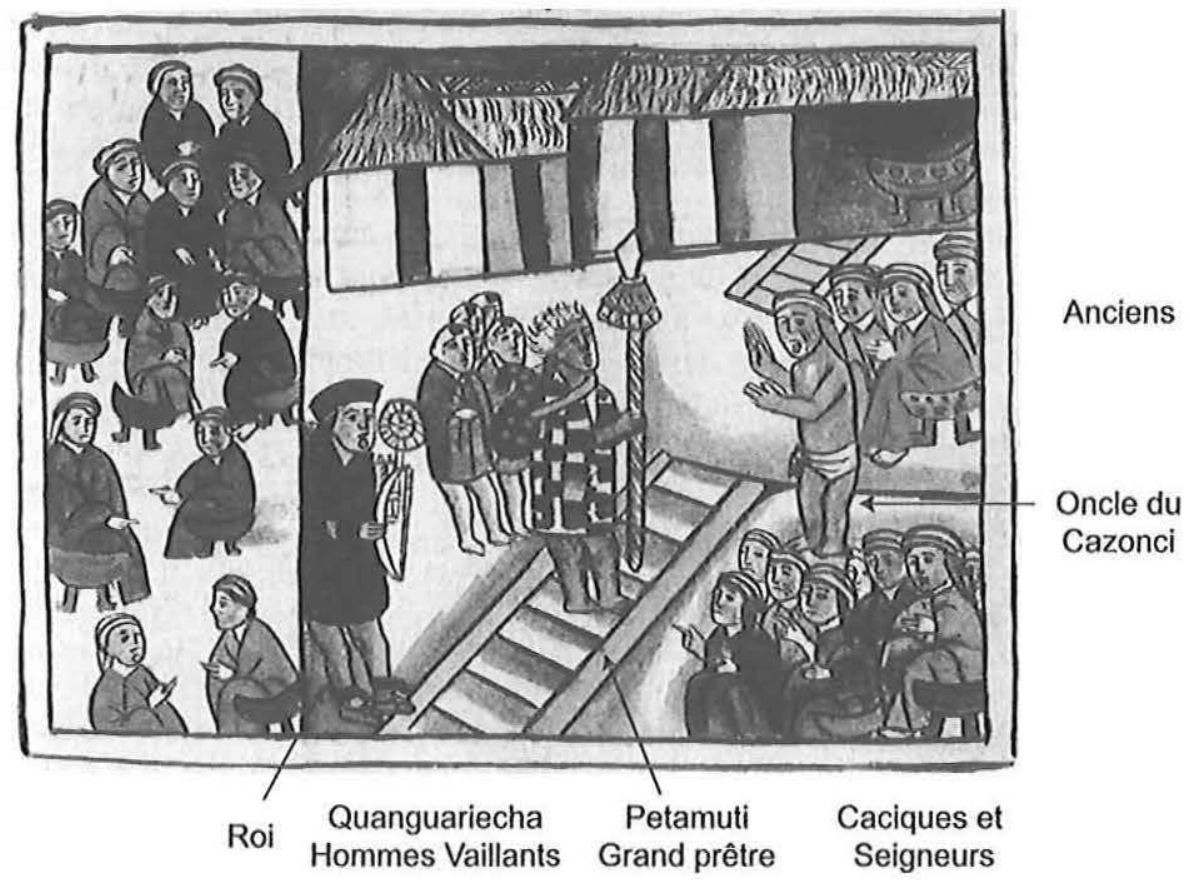

FIG. 8 - Les cérémonies d'investiture du cazonci (d'après la planche XL, in RM 2000).

royaume étaient réunis pour désigner le nouveau cazonci. Le fils du roi précédent, pressenti, prenait alors la parole pour proposer que la charge royale soit confiée à son oncle. Les échanges, au cours desquels les deux protagonistes refusaient successivement de devenir cazonci, duraient ainsi cinq jours, puis le fils acceptait enfin la charge royale. Il pouvait revêtir un costume qui est décrit en détail dans le texte.

poniase una guirnalda de cuero de tigre en la cabeza, y un carcax de cuero de tigre con sus flechas, o de otros animales, de colores, y un cuero de cuatro dedos en la muñeca, y unas manillas de cuero de venado con el pelo y uñas de venados en las piernas, que eran insinias de señor, y todos los señores se ponian de aquella manera, y partianse de su casa, y iban delante dél el sacerdote mayor... (RM 1997, p. 225)

La planche XL illustre cet épisode particulièrement important de la vie du royaume. Les détails du costume correspondent assez fidèlement à la description du texte, mais l'image apporte aussi de nombreuses précisions (Figure 8) : le roi porte un costume brun, qui tranche nettement avec la riche étoffe qu'arbore le grand prêtre à cette occasion. Ce vêtement court dont sont vêtus le nouveau 
cazonci et le petit groupe de quanguariecha qui se trouve à ses côtés aurait, en réalité, été taillé dans du cuir de cervidé, alors que, pour les jambes, étaient utilisés, selon le texte, des bandes de peau où le poil et les sabots avaient été préservés. Ces derniers ne sont cependant pas visibles sur l'illustration, peut-être à cause de leur taille.

En revanche, c'est de la fourrure de jaguar qui était employée pour réaliser la coiffe, le carquois et la bande de poignet du roi (Figure 9a). Les objets que le nouveau dirigeant porte dans ses mains ne sont pas décrits dans le texte ; ils ont, pourtant, une importance considérable. Dans sa main droite, comme dans beaucoup d'autres illustrations de la RM où il est représenté, le roi porte un arc qui constitue le symbole de son appartenance au groupe des guerriers et des chasseurs. Dans sa main gauche, il brandit au moins une flèche, mais aussi un objet particulier, formé d'un manche surmonté de deux cercles concentriques rayonnants. Cet objet est particulièrement intéressant et mérite qu'on s'y attarde. Il ressemble, en effet, aux tlachieloni ou itlachiaya, les " yeux » qui servent parfois d'attributs à Tezcatlipoca et à Xiuhtecuhtli dans l'iconographie du Haut Plateau Central, décrits par Sahagún comme des « instruments à voir » (Olivier 1997; Sahagún 1982 ; Seler 1908), et se retrouve également chez les Huichol actuels sous la forme des nierika (Kindl 2007). L'association avec Xiuhtecuhtli, le dieu du feu chez les Mexica, est évidemment la plus intéressante, au moins à première vue, puisque le cazonci est bien, dans l'ancienne société p'urhépecha, une incarnation de Curicaueri qui est, comme nous l'avons vu, d'abord le dieu du feu. La présence de cet appareil pourrait ainsi indiquer que, le jour de son investiture, le roi acquiert, au même titre que son costume, l'un des emblèmes de la divinité qu'il va désormais incarner ${ }^{5}$.

Lors de son investiture, le cazonci s'affiche comme appartenant au groupe des guerriers par son vêtement de peau et complète ses attributs par deux groupes d'objets caractéristiques : l'arc et la flèche, d'une part, l'appareil optique, d'autre part. Néanmoins, on constate que ce vêtement et l'un des attributs ne sont portés qu'au cours de cette cérémonie et que, si le cazonci dispose presque toujours de son arc à ses côtés, l'appareil optique disparaît et le costume lui-même est différent dans d'autres circonstances (Figure 9). Le dirigeant apparaît alors comme un dignitaire supérieur, comme le roi de tous les P'urhépecha, avec un costume d'étoffe qui lui couvre assez fréquemment les genoux, souvent de couleur jaune ou orangée et orné de motifs (raies, carreaux ou, plus rarement, un soleil dans le dos) en rouge, bleu et blanc/crème. Les coiffes sont elles aussi variées ; elles peuvent ressembler à des turbans de tissus, aux guirlandes de fils ou de végétaux mentionnées dans le texte ou encore à des bandeaux ornés de plumes multicolores, parmi lesquelles on reconnaît parfois les longues plumes de quetzal. En effet, les textiles fins et colorés, et bien évidemment les plumes, constituaient des matières précieuses, elles aussi chargées d'un symbolisme particulier. La splendeur de l'étoffe portée par le grand prêtre lors des cérémonies d'investiture ou de 

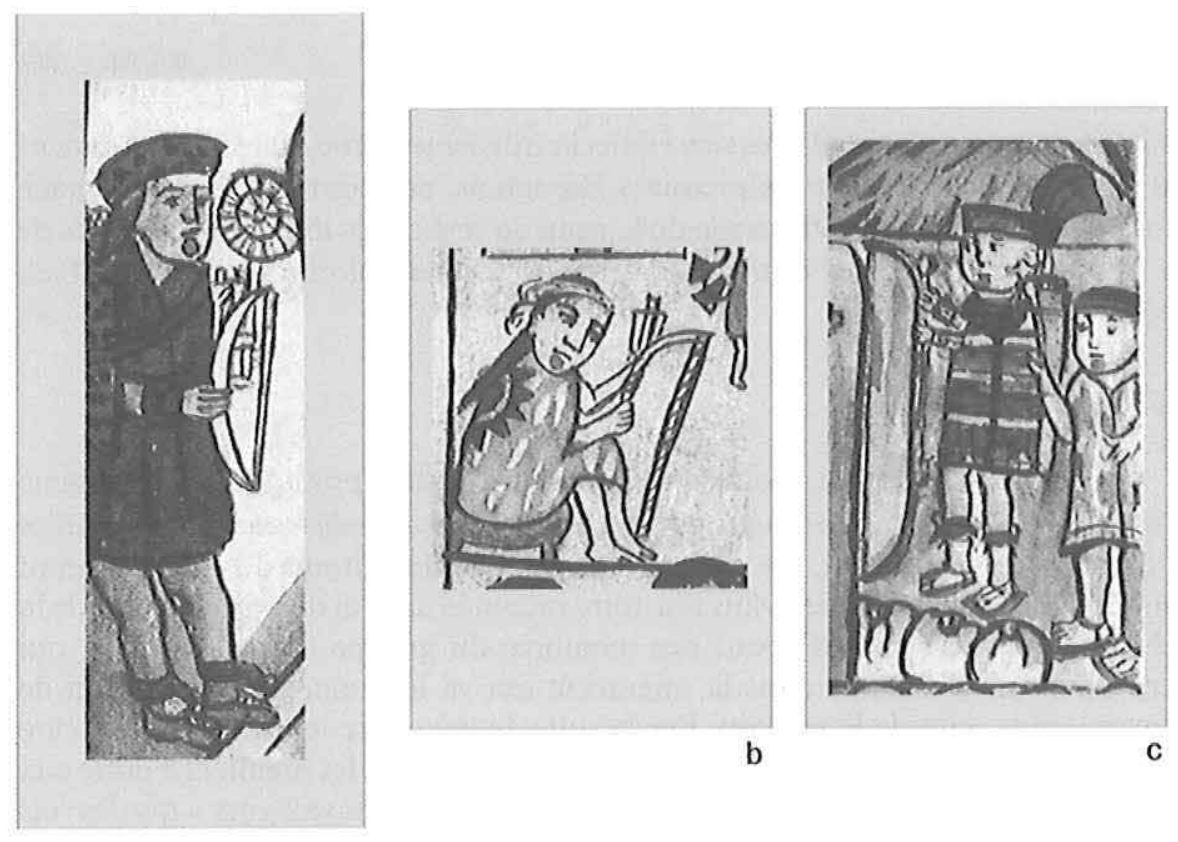

b

C

a
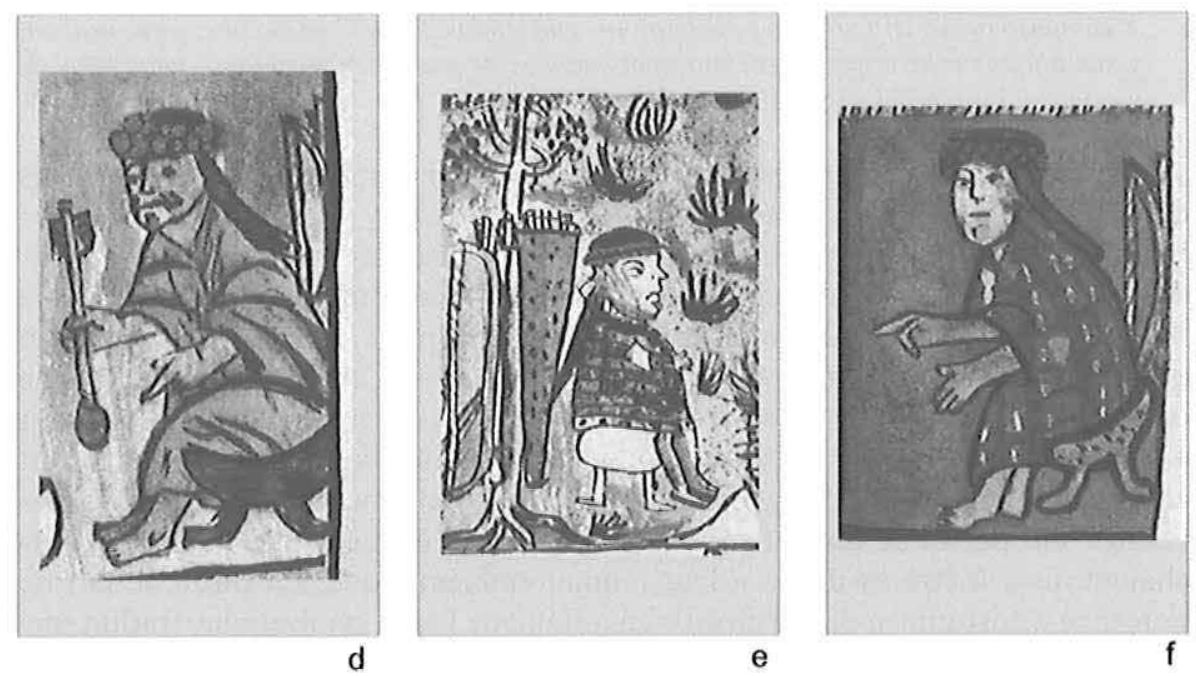

FIG. 9 - Exemples de costumes du cazonci : (a) investiture, vêtement court brun, coiffe et carquois en peau de jaguar, ornements de cheville en peau de cerf (d'après la planche XL, in RM 2000) ; (b) cérémonies suivant l'investiture, vêtement long jaune avec soleil rouge dans le dos (d'après la planche XLI, ibid.) ; (c) le cazonci Tzintzicha reçoit les Espagnols, costume rayé rouge et bleu, coiffe rouge et plumes de quetzal (d'après la planche XLIV, ibid.); (d) le cazonci Zuanga reçoit des cadeaux des Mexica, costume long orange et rouge, bandeau de trèfle (d'après la planche XLIII, ibid.) ; (e) le roi Taríacuri réalisant un rituel devant un feu sacré, vêtement avec lignes rouges et bleues (d'après la planche IX, ibid.) ; (f) Taríacuri ordonne la mort de son fils, vêtement court à raies rouges et blanches (d'après la planche XXV, ibid.). 
celle, identique, qu'arbore le cazonci dans la troisième partie du texte au moment où il s'apprête à recevoir les premiers Espagnols, en constitue un témoignage éloquent (Figure 9c). L'utilisation de la peau de cerf et des différents éléments de sa dépouille correspond donc à un langage et une volonté idéologique bien spécifiques.

\section{Le cerf et les guerriers}

Le cazonci porte, au moment de son investiture, pratiquement le même costume que les membres du groupe guerrier. Ces quanguariecha, ou « hommes vaillants ", constituaient, de fait, le premier cercle autour du roi, qui était lui-même issu de ce groupe. Dans l'histoire racontée dans la deuxième partie de la RM, les guerriers sont d'abord des membres du groupe uacusecha, ceux qui accompagnent Ticatame dans la migration qui va les guider vers le Bassin de Zacapu, puis celui de Patzcuaro. Par la suite, le lien entre le cazonci et son élite guerrière ne se dément pas : sous le règne du roi Tariacuri, les premiers d'entre eux sont ses frères, Aramen et Zetaco et c'est sur ces « hommes vaillants » que les rois vont s'appuyer pour les guerres de conquêtes. Le costume des hommes de guerre est clairement décrit dans la RM, notamment quand ils partent au combat.

Y compusiéronse, entizáronse y pusiéron sus guirnaldas de cuero en la cabeza que usaban, y sus aljabas a las espadas encima unos jubones de guerra, y pusiéronse unas uñas de venado en las piernas. (RM 1977, p. 39)

Le costume de ces quanguariecha peut être aussi observé sur les illustrations des planches V (Figures 4 et 5) et XL (Figure 8). On y voit le vêtement court brun, qui est apparemment taillé dans du cuir, les bandes de cuir nouées autour des poignets et celles où étaient préservés les sabots autour des chevilles. Le texte fait une nette différence entre les guirlandes de cuir pour la tête et les poignets et celles où pouvait être conservé le poil pour les jambes. Cette différence revêt une connotation symbolique indéniable, mais difficile à interpréter. Néanmoins le fait que le poil et les sabots aient été conservés pour les bandes de cuir qui sont enroulées aux chevilles des guerriers peut être vu comme si ces derniers « revêtaient » des pattes de cerf, et comme si, par leurs vêtements, les guerriers cherchaient ainsi à être assimilés à leur animal emblématique. En outre, le fait de porter ce " costume », de se travestir en animal de façon symbolique, traduit une volonté d'afficher une identité et une position hiérarchique particulières, à l'instar des chevaliers aigles et jaguars mexica quand ils revêtaient leurs costumes distinctifs sur le champ de bataille. Sur un autre plan, on pourrait voir aussi dans le choix d'arborer ce costume la manifestation de l'idée - largement répandue dans l'ensemble de la Mésoamérique - selon laquelle le chasseur prend la place de la proie et le sacrificateur celle de la victime : le chasseur devient le cerf et le guerrier; un sacrifié. Cette interprétation pourrait ainsi renvoyer aux parallèles évoqués 
précédemment entre chasse et guerre, le guerrier étant potentiellement une victime pour les sacrifices.

$\mathrm{Si}$ les guerriers se distinguent par leurs costumes, les planches illustrent également le fait qu'ils avaient une coiffure particulière. En effet, on l'a vu, la tonsure était de rigueur chez les quanguariecha - ainsi que chez les porteurs de dieux (Figure 10) -, et il ne faut pas oublier que l'un des noms qui était donné aux Tarasques lors de la Conquête, "los rapados ", fait référence à leur façon de se couper les cheveux qui aurait pu être l'apanage des guerriers. Chez les voisins mexica, la tonsure, totale ou partielle, du crâne pouvait être un signe de vaillance à la guerre et constituait un symbole de force et de vitalité associé au pouvoir du souverain et à sa capacité à alimenter les sacrifices humains (Olivier 1997, p. 43). Les cheveux avaient, selon López Austin (1989, p. 182), des fonctions magiques liées à leur proximité avec l'emplacement du tonalli; ils étaient utilisés, comme cela a déjà été indiqué, dans des rituels liés à la guerre et aux guerriers. On retrouve une symbolique des cheveux comparable chez les Tarasques, repérable notamment dans les événements qui avaient lieu lors des cérémonies de la fête de Sicuindiro, dont la traduction du nom " où s'enlève la peau » semble également faire référence à la Tlacaxipeualiztli mexica (Seler 2000). Néanmoins, c'est lors de la fête de Cuingo qu'était honoré Xipe Totec et qu'étaient consommés les pains de Tuitze, comme cela a été évoqué précédemment ${ }^{6}$. La fête de Sicuindiro, l'une des mieux décrites dans la RM, se déroulait à Zinapecuaro et Araro en l'honneur de la déesse mère Cuerauáhperi, probablement autour du 15 septembre de chaque année. Parmi les officiants principaux, on trouvait les hauiripicipecha (« ceux qui quittent aux autres leurs cheveux ») qui, après avoir jeûné puis procédé aux sacrifices humains, couraient après les hommes et les femmes pour leur couper les cheveux à l'aide de lames d'obsidienne (Espejel Carvajal 2004, p. 160). Mais Curicaueri était aussi honoré lors de cette fête, dans une cérémonie dédiée à la guerre ; c'est notamment à cette occasion qu'avaient lieu les rites d'initiation des jeunes guerriers qui étaient rasés à ce moment-là (Corona Nuñez 1946, p. 29). Les cheveux récoltés étaient ensuite trempés dans le sang des victimes sacrificielles et jetés dans les bûchers sacrés. On voit ici comment les rituels d'initiation des jeunes guerriers - notamment par la tonsure et l'offrande de cheveux, élément du corps déterminant pour la vaillance à la guerre - renvoient à leur fonction de pourvoyeurs en victimes pour les sacrifices.

Le rôle symbolique joué par la coiffure et par les cheveux des guerriers tarasques incite à revenir sur la description qui est donnée de la physionomie du cerf mythique Tuitze. En effet, comment interpréter, dans ce cadre, un détail qui lui donne sa spécificité, c'est-à-dire les crins qui sont apposés sur sa nuque? L'emplacement mentionné ( "la cerviz ») stipule bien qu'il s'agit de la tête de l'animal, et non de son encolure. Il est ainsi probable que, plus qu'une crinière, il faille y voir une touffe de crins semblable aux cheveux, dont le symbolisme serait essentiel puisqu'il conférerait à cet être mythique des pouvoirs spécifiques dans 


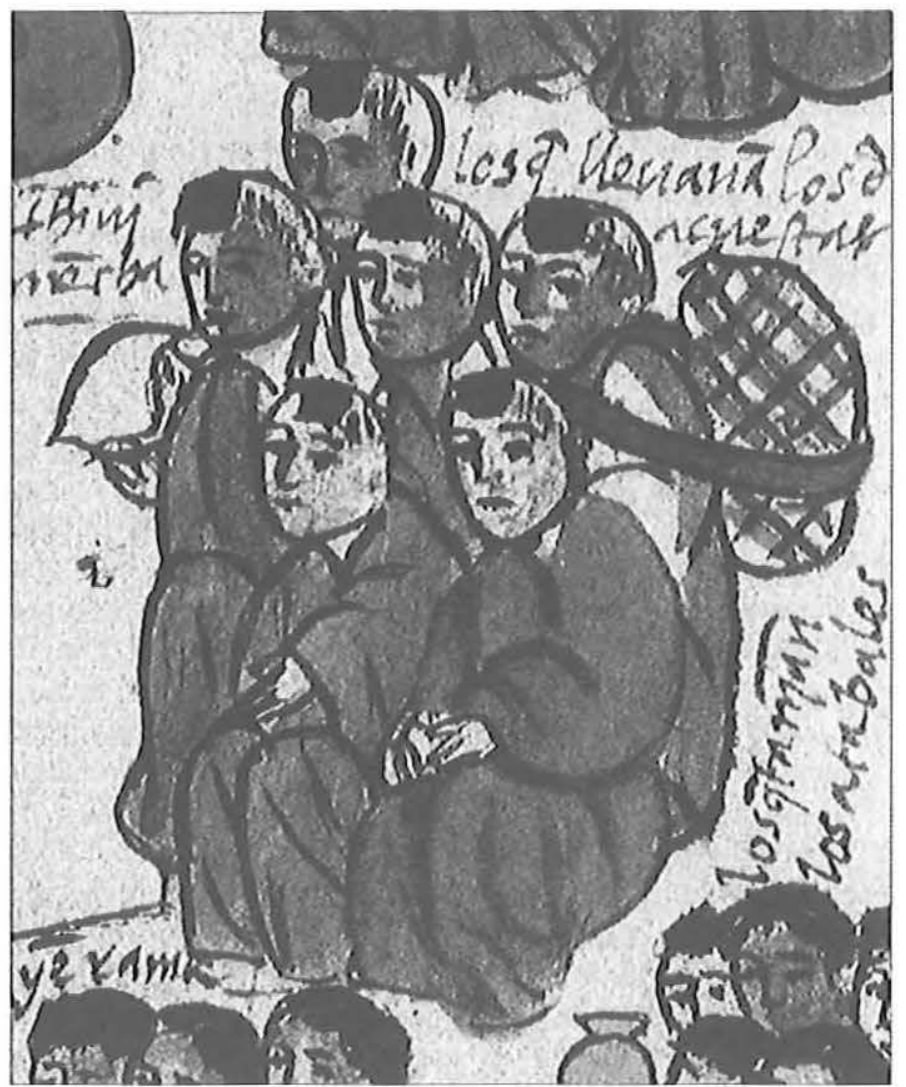

Fig. 10 - Les porteurs de dieux et leurs tonsures (d'après la planche XXX, in RM 2000).

les activités guerrières. Par ailleurs, cette « chevelure » du cerf tend aussi à l'« anthropomorphiser » et à le rapprocher du groupe des guerriers.

\section{COMMENTAIRES FINAUX}

Au terme de cette enquête sur la place du cerf dans l'idéologie des anciens P'urhépecha, il devient possible de se demander si cette position est vraiment caractéristique du Michoacan, ou bien si on la retrouve à une échelle plus vaste qui pourrait englober le Centre et l'Occident du Mexique. Par ailleurs, on peut s'interroger sur les correspondances que l'on pourrait dresser à l'échelle mésoaméricaine en examinant la place occupée par les cervidés dans un ensemble de croyances beaucoup plus vaste sur un plan géographique et culturel. 
Comme nous l'avons vu, chez les Tarasques, le cerf occupe une position complexe qui a, de plus, probablement évolué avec le temps (Pollard 1991). Cette évolution est peut-être perceptible dans le rôle qu'ont les cervidés dans les deux parties de la RM ; leur utilisation se transforme en effet nettement entre la période qui a précédé l'unification du royaume, dans un contexte de revendications idéologiques de la part d'une élite luttant pour asseoir son hégémonie, et le moment où, environ un siècle plus tard, peut se mettre en place un véritable panthéon.

Les sources disponibles indiquent d'abord que l'animal était considéré comme la proie privilégiée à la chasse, mais il joue surtout un rôle éminent dans les rituels, notamment dans un cadre sacrificiel. Dans la deuxième partie de la RM, le cerf est mentionné comme une nourriture pour les dieux, sans qu'il soit précisé toutefois si cette offrande était dédiée à une divinité particulière et si elle était consommée sous forme de viande à l'occasion de fêtes spécifiques. C'est par la chasse que les hommes se procurent cet aliment : on capture l'animal pour le sacrifier devant un temple, comme on capture des hommes à la guerre pour les offrir aux divinités. Le sang humain peut remplacer le sang du cerf, la guerre se déroule comme une chasse, et la chair humaine peut remplacer la chair de l'animal dans les banquets. Ainsi, une double homologie se dessine dans laquelle la chasse et la guerre sont les moyens utilisés pour se procurer des cerfs et/ou des êtres humains. Cette homologie entre l'homme et le cerf pourrait, par ailleurs, se retrouver dans le fait que les os longs des deux espèces auraient pu constituer les supports pour la fabrication des mêmes instruments rituels.

En Mésoamérique précolombienne, l'utilisation des cervidés comme offrandes sacrificielles lors de certains rituels et l'homologie homme-cerf sont bien connues et des réminiscences de cette tradition sont d'ailleurs encore perceptibles dans les rituels de certaines populations actuelles. Ces pratiques, qui traduisent le principe général de l'ambivalence, présente dans la pensée mésoaméricaine, entre le chasseur et sa proie et entre le sacrifié et le sacrificateur, se retrouvent notamment chez les Mayas et chez les Aztèques. Les sacrifices de cerfs sont mentionnés dans les textes tardifs (Codex Borgia et Codex Madrid) et représentés dans l'iconographie, par exemple chez les Mayas classiques. Dans son étude sur le sujet, Pohl (1981) mentionne un certain nombre de pièces archéologiques où le sacrifice du cerf est clairement figuré. Dans la zone maya, le sacrifice du cerf semble associé à des rites agraires, au maïs et à la pluie, mais il aurait été aussi intimement lié à la noblesse et pratiqué au moment des rites d'accession au pouvoir (Pohl 1981, p. 521). Les comparaisons entre les pratiques tarasques et aztèques sont encore plus éloquentes. En effet, chez les Mexica, des chasses rituelles étaient organisées lors de certaines fêtes afin de fournir des cerfs pour les sacrifices ; la guerre elle-même était considérée comme une chasse (Graulich 1997). Au sein des sociétés actuelles, l'offrande de cerf demeure connue dans certaines sociétés comme chez les Tlapanèques (Dehouve 2005) ou dans le 
Guerrero (Neff 2005). Mais les comparaisons les plus fructueuses peuvent être dressées avec les groupes qui vivent au nord-ouest de l'État du Michoacan dans la Sierra Madre occidentale (États de Jalisco et Nayarit), qui revendiquent d'ailleurs une origine "chichimèque», et parmi lesquels figure notamment un groupe particulièrement bien étudié, les Huichol. L'importance du cerf dans les croyances et les rituels de cette société semble tout à fait exceptionnelle encore à l'époque moderne et une riche mythologie est associée à cet animal (Lumholtz 1986). Malgré la disparition progressive des cervidés dans les montagnes du Nayarit, la chasse au cerf demeure essentielle, puisque « aucune fête du cycle cosmologique et politique - donc comportant des sacrifices - ne peut avoir lieu sans une chasse préalable » (Lemaistre et Kindl 1999, p. 212 ; Lemaistre 1991). Le cerf se livre parfois lui-même aux hommes dans un acte d'autosacrifice ${ }^{7}$ et l'idée de sacrifice lui est directement associée. Comme prise de chasse, le cerf peut présenter, chez les Cora voisins, une association avec Vénus, étoile du soir, et les étoiles en général (Preuss 1912; Neurath 2004). Ces différents aspects nous renvoient à plusieurs acceptions symboliques évoquées chez les Tarasques préhispaniques. Parmi les nombreuses figures du cerf chez les Huichol domine celle de l'ancêtre civilisateur Tamatsi Kauyumari, Notre Frère Aîné. Cet ancêtre a émergé, pour la première fois, de l'océan Pacifique et fut le premier chamane. Il est la figure qui permet aux hommes de communiquer avec les ancêtres déifiés à travers les rêves, la consommation de peyotl ou la fabrication de certains objets selon des règles précises (Kindl 2005 et 2007). Comme le cerf mythique des anciens P'urhépecha, il est intimement lié au cycle solaire et, plus spécifiquement, au soleil levant, à l'est, à la saison sèche, et il constitue un symbole associé au pouvoir (Kindl 2005; Lemaistre 1991). Ailleurs en Mésoamérique, le cerf représente en général une figure prééminente dans le monde surnaturel - peut-être autant que le jaguar -, ce qui aurait, au moins au Postclassique, généré des interdits alimentaires (Pohl 1981). Chez les Mayas, il semble associé à la pluie, même si le Codex de Madrid évoque également un lien avec le dieu de l'inframonde (ibid.) et que le Popol Vuh lui fait aussi jouer un rôle majeur dans les mythes d'origine, où il est associé au soleil (Benson 1997).

Si les utilisations rituelles des cervidés chez les anciens P'urhépecha s'insèrent dans un cadre global mésoaméricain, l'originalité de leur position tient plutôt au rôle qui leur est réservé par l'élite dirigeante, comme chez les Huichol contemporains. En effet, cet animal était aussi utilisé par l'élite guerrière et par le roi comme un élément identitaire, pour exprimer ainsi une revendication précise sur l'origine du royaume (Faugère K. 1998) et pour rappeler la spécificité de leur position sociale. À travers lui, c'est leur origine exogène de chasseurs et la position dominante de Curicaueri, leur dieu tutélaire, qui sont réaffirmées. Par la guerre, le roi et ses guerriers ont avant tout le devoir de maintenir la domination de Curicaueri sur un territoire étendu et un peuple nombreux, et d'alimenter les sacrifices en l'honneur des dieux. C'est donc également grâce à sa qualité d'avatar 
de la divinité solaire que le cerf est lié avec le roi et l'élite guerrière, mais c'est aussi en tant qu'ancêtre des hommes. En ce sens, la représentation de l'appareil optique exhibé par le cazonci au moment de son investiture - qui est aussi l'un des attributs de Tezcatlipoca sur le Haut Plateau central - pourrait renvoyer à cet aspect lié à la communication avec les ancêtres et à la divination. Comme dans plusieurs civilisations mésoaméricaines, un rôle particulier serait attribué par les Tarasques au cerf au moment des rites d'intronisation, mais cette intervention se situe bien évidemment à des niveaux très différents. Nous avons largement évoqué la forme sous laquelle les cervidés étaient utilisés au moment de l'investiture du cazonci et, par ailleurs, les parallèles qui pouvaient être établis dans le déroulement de cette cérémonie bien particulière chez les Tarasques et chez les Mexica si l'on accepte, bien entendu, de considérer que les sources ethnohistoriques ne se sont pas influencées mutuellement.

Cet ensemble de données montre que les cervidés jouaient un rôle éminent dans la pensée mythique mésoaméricaine, dans les croyances et dans les rituels, mais les comparaisons indiquent aussi qu'une réelle parenté se dégage, pour l'époque préhispanique, avec les Aztèques. Sur un plan historique, cette parenté n'est pas réellement surprenante, puisque les deux civilisations partagent des trajectoires parallèles. Cependant, l'élite du Michoacan avait conservé aux cervidés un rôle majeur, alors que le système de croyances des Mexica avait évolué différemment, dans le sens de ce qu'on considère comme une complexification croissante. Néanmoins les exemples contemporains, et notamment celui des Huichol, montrent combien une mythologie extrêmement riche et complexe peut aussi être associée à un seul animal. *

* Manuscrit reçu en janvier 2007, accepté pour publication en mai 2008.

\section{NOTES}

1. À l'époque préhispanique, trois variétés de cervidés se répartissaient sur le territoire mésoaméricain. Le cerf à queue blanche (Odocoileus virginianus) était le plus commun, notamment dans les hautes terres, alors que les deux autres connaissaient une diffusion moindre, le cerf mulet se localisant surtout dans le nord du Mexique et le mazama dans les zones au climat tropical humide. Tous ces cervidés avaient des tailles bien inférieures au cerf européen.

2. En dehors du codex Plancarte, mais qui reste difficile à interpréter.

3. Le chiffre cinq aurait eu chez les Tarasques une forte connotation symbolique, associée au culte des points cardinaux et du centre (Corona Nuñez 1946; Pollard 1993). Il se retrouve dans d'autres offrandes, notamment celles de flèches.

4. Bien que le contexte de découverte ne soit pas connu, les différentes pièces réunies dans cet ensemble faisaient partie de la même offirande : en effet, les dimensions sont très homogènes entre grelots et masques et, par ailleurs, la matière première et les techniques de fabrication (à la cire perdue) semblent en tous points identiques. Ces pendentifs sont de petite taille et mesurent entre $2 \mathrm{et} 2,5 \mathrm{~cm}$ de longueur approximativement pour une largeur à peu près identique. Cet ensemble appartenait à l'ancienne collection Guy Joussemet. 
5. L'instrument à voir est également un attribut de Tezcatlipoca qui peut être observé dans plusieurs représentations du dieu dans les codex aztèques, par exemple dans le Codex Tudela, le Codex Magliabechiano ou encore le Codex Florentino (Olivier 1997), où l'on peut voir que le dieu tient cet instrument dans sa main gauche. Durán (1995) le décrit comme étant un émouchoir formé d'un miroir orné de plumes bleues, vertes et jaunes, mais Olivier (1997, p. 68) identifie cet éventail comme étant en réalité l'appareil optique évoqué précédemment. Parmi ses multiples aspects et fonctions, Tezcatlipoca aurait joué un rôle clef chez les Aztèques au moment de l'investiture du nouveau tlatoani. En effet, il était clairement invoqué dans les échanges de discours prononcés au cours des cérémonies d'investiture : un grand prêtre ou un grand dignitaire mettait en garde le seigneur pressenti et lui faisait toutes sortes d'avertissements afin que le «dieu moqueur » ne se détourne pas du nouveau dirigeant. Ce dernier devait lui-même solliciter son aide et se mettre sous sa protection. Des parallèles peuvent ainsi être dressés à plusieurs niveaux entre les rituels d'intronisation du tlatoani mexica et ceux du cazonci tarasque, au point que l'on peut se demander si ces parallélismes ne sont pas plutôt dus à ce que les textes de la première moitié du xvi siècle ont servi pour la rédaction d'ouvrages plus tardifs, comme le suggère Espejel Carvajal (2004, p. 287).

6. Seler $(2000$, p. 230$)$ souligne l'étroite relation entre les deux divinités agraires, la mère des dieux et Xipe Totec, proximité qui aurait, selon lui, disparu depuis longtemps dans les cultes des Mexica.

7. À cette occasion, il se transforme en peyotl.

\section{RÉFÉRENCES CITÉES}

Acosta Jorge

1939 « Exploraciones arqueológicas realizadas en el Estado de Michoacán entre los años 1937 y 1938 », Revista Mexicana de Estudios Antropológicos, III (2), pp. 85-99.

Benson Elizabeth P.

1997 Birds and beasts of Ancient Latin America, University Press of Florida, Gainesville.

Codex Borgia

19633 vol., éd. Eduard Seler, Fondo de Cultura Económica, Mexico.

\section{Codex Florentino}

1979 El manuscrito 218-220 de la colección Palatina de la Biblioteca Medicea Laurenziana, éd. facs, 3 vol., Giunti Barbéra/Archivo General de la Nación, Florencia/Mexico.

\section{Codex Madrid}

1977 in Códices mayas, J. Antonio Villacorta et Carlos A. Villacorta (éds), Tipografía nacional, Guatemala.

\section{Codex Magliabechiano}

1996 éd. Ferdinand Anders et Maarten Jansen, Akademische Druck-u. Verlagsanstalt/Fondo de Cultura Económica, Graz/Mexico.

\section{Codex Tudela}

1980 éd. José Tudela de la Orden, Ediciones Cultura Hispánica del Instituto de Cooperación Iberoamericano, Madrid. 


\section{CORONA NuÑEz José}

1946 "La religión de los tarascos », Anales del Museo Michoacano, 4, segunda época, Morelia, pp. 13-38.

1977 Necesaria interpretación de la Relación de Michoacán o Códice Escurialense. Relación de las Ceremonias y ritos y población y gobierno de los Indios de la provincia de Michoacán : $V-X X$, reproducción facsímil del Ms. 9.IV.5 de El Escorial, transcripción de José Tudela, estudio de José Corona Nuñez, Balsal Ed., Morelia.

\section{Darras Véronique}

1998 «La obsidiana en la Relación de Michoacán y en la realidad arqueológica : del símbolo al uso y del uso de un símbolo ", in Véronique Darras (éd.), Génesis, culturas y espacios en Michoacán, CEMCA, Mexico, pp. 61-88.

\section{Dehouve Danièle}

2005 "Les rituels cynégétiques des Indiens mexicains », in Isabelle Sidéra (éd.), La chasse. Pratiques sociales et symboliques, De Boccard Éd., Paris, pp. 195-204.

Di PEso Charles

1974 "Casas Grandes, a fallen trading center of the Gran Chichimeca », The Amerind Foundation, 1-3 (9), Northland Press, Flagstaft.

DURÁN Fray Diego

1995 Historia de las Indias de Nueva España e islas de Tierra Firme, 2 vol., éd. José Rubén Romero et Rosa Carmelo, Cien de México/CONACULTA, Mexico.

Espejel Carvajal Claudia

2004 Voces, lugares y tiempos : claves para comprender la Relación de Michoacán, tesis de doctorado, El Colegio de Michoacán, Zamora.

FAUGÈRE K. Brigitte

1996 "Entre Zacapu y Río Lerma : culturas en una zona fronteriza », Cuadernos de Estudios Michoacanos, 7, CEMCA, Mexico.

1997 «Las representaciones rupestres del centro-norte de Michoacán », Cuadernos de Estudios Michoacanos, 8, CEMCA, Mexico.

1998 «Venados y hogares sagrados en la Relación de Michoacán : reivindicación nórdica y construcción del Estado en los pueblos tarascos ", in Véronique Darras (éd.), Génesis, culturas y espacios en Michoacán, CEMCA, Mexico, pp. 89-99.

GALI Ramón

1946 "Arqueología de Tzintzuntzan, temporada », Anales del Museo Michoacano, segunda época, 4.

Graulich Michel

1997 "Chasse et sacrifice humain chez les Aztèques 》, Académie Royale des Sciences d'Outre-mer: Bulletin des Sciences, 43 (4), Bruxelles, pp. 433-445. 


\section{Hurtado Mendoza Franciso}

1986 La religión prehispánica de los Purhépecha : un testimonio del pueblo tarasco, [s. n.], Morelia.

KINDL Olivia

2005 "L'art du nierika chez les Huichol du Mexique. Un "instrument pour voir" " , in Michèle Coquet, Brigitte Derlon et Monique Jeudy-Ballini (éds), Les cultures à l'cuvre. Rencontres en art, Biro éditeur/Éditions de la Maison des sciences de l'homme, Paris, pp. 225-248.

2007 Le nierika des Huichol: un " art de voir », thèse de doctorat, Université de Paris X, Nanterre.

Le Clezio Jean-Marie Gustave

2000 "Universalidad de la Relación de Michoacán », in Relación de Michoacán. Estudios introductorios, El Colegio de Michoacán, Zamora, pp. 107-120.

\section{Lemaistre Denis}

1991 "Le cerf-peyotl et le cerf-maïs : la chasse, rituel formateur de la "trinité" huichole ", Journal de la Société des Américanistes, 77, pp. 27-43.

Lemaistre Denis et Olivia KIndL

1999 «La semaine sainte huichole de Tateikie : rituel solaire et légitimation du pouvoir par les sacrifices ", Journal de la Société des Américanistes, 85, pp. 175-214.

López Austin Alfredo

1989 Cuerpo humano e ideología, UNAM, Mexico.

Lumholtz Carl

1986 El arte simbólico y decorativo de los huicholes, Instituto nacional indigenista, Mexico.

Michelet Dominique

1989 " Histoire, mythe et apologue : notes de lecture sur la seconde partie de la Relation [...] de Michoacan ", in Dominique Michelet (éd.), Enquêtes sur l'Amérique Moyenne. Mélanges offerts à G. Stresser Péan, INAH/Consejo Nacional para la cultura y las artes/CEMCA, Mexico, pp. 105-113. « Topografía y prospección sistemática de los grandes asentamientos del Malpaís de Zacapu : claves para un acercamiento a las realidades sociopolíticas ", in Véronique Darras (éd.), Génesis, culturas y espacios en Michoacán, CEMCA, Mexico, pp. 47-60.

Migeon Gérald

1990 Archéologie en pays tarasque. Structure de l'habitat et ethnopréhistoire des habitats tarasques de la région de Zacapa (Michoacan, Mexique) au Postclassique Récent, thèse de doctorat, Université de Paris I-PanthéonSorbonne, Paris.

NeFF Françoise

2005 Mouvement et intensité dans la pensée indienne. Mythes et rituels de l'État de Guerrero, Mexique, thèse de doctorat, Université de Paris X, Nanterre. 
Neurath Johanes

2004 "El doble personaje del planeta Venus en las religiones indigenas del Gran Nayar : mitología, ritual agrícola y sacrificio », Journal de la Société des Américanistes, 90 (1), pp. 93-119.

OLivier Guilhem

1995 «Les paquets sacrés ou la mémoire cachée des Indiens du Mexique central », Journal de la Société des Américanistes, 81, pp. 105-141.

1997 Moqueries et métamorphoses d'un dieu aztèque. Tezcatlipoca, le "Seigneur au miroir fumant », Institut d'Ethnologie, Paris.

PEREIRA Grégory

2005 "The utilization of grooved human bones : a reanalysis of artificially modified human bones excavated by Carl Lumholtz at Zacapu, Michoacan, Mexico ", Latin American Antiquity, 16 (3), pp. 293-312.

2007 «Le destin post-mortem du guerrier : pratiques funéraires et statut social dans le Michoacan ancien, Mexique ", in Luc Baray, Patrice Brun et Alain Testart (éds), Actes du colloque : pratiques funéraires et société, Éditions universitaires de Dijon, coll. "Art, archéologie et patrimoine », Dijon, pp. $370-388$.

\section{Pérez Martinez Héron}

2000 "El arte literario de la Relación de Michoacán», in Relación de Michoacán. Estudios introductorios, El Colegio de Michoacán, Zamora, pp. 79-106.

PoHL Mary

1981 «Ritual continuity and transformation in Mesoamerica. Reconstructing the ancient maya cuch ceremony ", American Antiquity, 46 (3), pp. 513529 .

Pollard Helen

1991 "The construction of ideology in the emergence of Prehispanic Tarascan state ", Ancient Mesoamerica, 2 (2), pp. 167-179.

1993 Tariacuri's Legacy. The Prehispanic Tarascan State, Oklahoma University Press, Norman.

Preuss Konrad T.

1912 Die Nayarit-Expedition. 1. Die religion der Cora-Indianer in texten nebst wörterbuch Cora-Deutsh, B. G. Teubner, Leipzig.

Relación de las ceremonias y ritos y población y gobierno de los Indios de la provincia de Michoacán

1977 reproducción facsímil del Ms. 9.IV.5 de El Escorial, transcripción de José Tudela, estudio de José Corona Nuñez, Balsal Ed., Morelia.

\section{Relación de Michoacán}

2000 por José de Alcalá, Moisés Franco Mendoza (éd.), El Colegio de Michoacán/Gobierno del Estado de Michoacán, Zamora. 


\section{ROSKAMP Hans}

2000 «El Carari indígena y las láminas de la Relación de Michoacán : un acercamiento », in Relación de Michoacán. Estudios introductorios, El Colegio de Michoacán, Zamora, pp. 79-106.

SAHAGÚN Fray Bernardino (de)

1982 Historia General de las cosas de la Nueva Espana, Ed. Porrúa, Mexico.

Seler Eduard

1908 "Costumes et attributs des divinités du Mexique selon P. Sahagún », Journal de la Société des Américanistes, 5 (2), pp. 163-220.

2000 "Los antiguos habitantes de Michoacán ", in Relación de Michoacán. Estudios introductorios, El Colegio de Michoacán, Zamora, pp. 139-234.

Terán Elizondo María Isabel

2000 "Elementos mítico-simbólicos », in Relación de Michoacán. Estudios introductorios, El Colegio de Michoacán, Zamora, pp. 285-299.

WILLIAMS Eduardo

1992 Las piedras sagradas. Escultura prehispánica del Occidente de México, El Colegio de Michoacán, Zamora. 